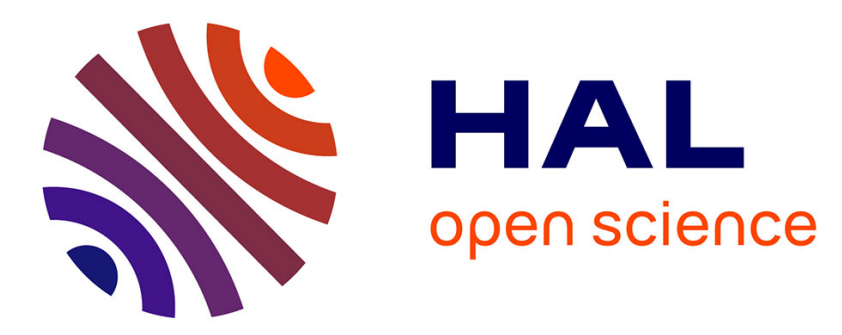

\title{
Critical Role of Interfacial Diffusion and Diffuse Interphases Formed in Multi-Micro-/Nanolayered Polymer Films Based on Poly(vinylidene fluoride) and Poly(methyl methacrylate)
}

Bo Lu, Khalid Dr. Lamnawar, Abderrahim Maazouz, Guillaume Sudre

\section{To cite this version:}

Bo Lu, Khalid Dr. Lamnawar, Abderrahim Maazouz, Guillaume Sudre. Critical Role of Interfacial Diffusion and Diffuse Interphases Formed in Multi-Micro-/Nanolayered Polymer Films Based on Poly(vinylidene fluoride) and Poly(methyl methacrylate). ACS Applied Materials \& Interfaces, 2018, 10 (34), pp.29019-29037. 10.1021/acsami.8b09064 . hal-02010566

\author{
HAL Id: hal-02010566 \\ https://hal.science/hal-02010566
}

Submitted on 5 Mar 2020

HAL is a multi-disciplinary open access archive for the deposit and dissemination of scientific research documents, whether they are published or not. The documents may come from teaching and research institutions in France or abroad, or from public or private research centers.
L'archive ouverte pluridisciplinaire HAL, est destinée au dépôt et à la diffusion de documents scientifiques de niveau recherche, publiés ou non, émanant des établissements d'enseignement et de recherche français ou étrangers, des laboratoires publics ou privés. 
Critical role of interfacial diffusion and diffuse interphases formed in multi micro-/nanolayered polymer films based on poly(vinylidene fluoride) and poly(methyl methacrylate)

Bo Lu, ${ }^{\dagger}$ Khalid Lamnawar, ${ }^{*,+}$ Abderrahim Maazouz, ${ }^{\dagger, \S}$ and Guillaume Sudre ${ }^{\ddagger}$

\footnotetext{
† Université de Lyon, CNRS, UMR 5223, Ingénierie des Matériaux Polymères, INSA Lyon, F-69621,Villeurbanne, France

† Université de Lyon, CNRS, UMR 5223, Ingénierie des Matériaux Polymères, Université Claude Bernard Lyon 1, F-69622, Villeurbanne, France

${ }^{\S}$ Hassan II Academy of Science and Technology, 10100 Rabat, Morocco
}

Published in ACS Applied Materials and Interfaces:

Critical Role of Interfacial Diffusion and Diffuse Interphases Formed in Multi-Micro-/Nanolayered Polymer Films Based on Poly(vinylidene fluoride) and Poly(methyl methacrylate). Lu B., Lamnawar K., Maazouz A., Sudre G., ACS Applied Materials \& Interfaces, 2018, 10(34), 29019-29037.

Link to the publisher version: http://dx.doi.org/10.1021/acsami.8b09064

Creative Commons Attribution Non-Commercial No Derivatives License 


\begin{abstract}
It is known that the macroscopic properties of multilayer polymer films are largely dominated by the diffuse interphase formed via interfacial diffusion between neighboring layers. However, not much is known about the origin of this effect. In this work, we reveal the role of interfacial diffusion and the diffuse interphase development in multilayer polymer films, based on a compatible poly(vinylidene fluoride)/poly(methyl methacrylate) system fabricated by forced assembly micro-/nanolayer coextrusion. Interestingly, the layer morphology is found to prevail in all investigated multilayer films, even for the nanolayered system where the interdiffusion is substantial. It is also demonstrated that, in the presence of macromolecular and geometrical confinements, interfacial diffusion significantly alters the crystalline morphology and microstructure of the resulting micro-/nanolayered films, which leads to quantitatively different dielectric and rheological properties. More importantly, the combination of dielectric relaxation spectroscopy and energy dispersive X-ray analysis further reveals that multiple diffuse interphases with various length scales exist in the multilayer structures. The presence of these multiple interphases is explained in terms of a proposed physical picture for the interdiffusion of fast-mode mechanism occurring in coextrusion process, and their length scales (i.e., interphase thicknesses) are further mapped quantitatively. These findings provide new insights into the effects of interfacial diffusion and diffuse interphases, toward tailoring interfaces/interphases in micro-/nanolayered polymer structures and for their advanced applications.
\end{abstract}

Keywords: Forced assembly coextrusion, multilayer films, interfacial diffusion, interface, interphase, structure, properties 


\section{Introduction}

Nowadays, flexible and light-weight functional multilayer films are increasingly used in application areas such as printable electronics, capacitors, energy, nano-medicine, automotive engineering and construction. Among the common techniques fabricating multilayer polymer films, multilayer coextrusion has been a versatile technology and attracted tremendous interest over the past two decades, mainly due to the enhanced dielectric, optical, mechanical, and gas barrier properties of the multilayered products. ${ }^{1-5}$ Different from the other traditional methods based on molecular assembly concepts, such as layer-by-layer assembly, ${ }^{6}$ multilayer coextrusion termed "forced assembly" is a top-down approach and capable of manufacturing industrially multilayer films with thousands of alternating layers at the lower environmental and pecuniary costs. ${ }^{1,3}$ Using a layer multiplication concept, this technology controls the layer architecture/morphology and layer thickness from micro- down to nanoscale towards the target properties. ${ }^{7,8}$ The markedly improved macroscopic properties in the coextruded multilayer films are generally attributed to the dominating interface/interphase and/or confinement effects. ${ }^{2,9}$

Considerable efforts have been made to prepare multilayered films with a great number of interfaces by the combination of alternating layers of immiscible or partially miscible polymer pairs, such as polystyrene (PS)/ poly(methyl methacrylate) (PMMA), ${ }^{10,}{ }^{11}$ polycarbonate (PC)/PMMA, ${ }^{12}$ PC/poly(vinylidene fluoride) (PVDF), ${ }^{13,14}$ polysulfone/PVDF, ${ }^{15,16}$ and PS/polycaprolatone (PCL), ${ }^{17}$ etc. Using laminar flow conditions to combine these polymer pairs with short contact times, one can greatly minimize the localized mixing of polymer segments at the polymer-polymer boundary (interface) and thus produce multilayer films and composites having a large quantity of interfaces. Although the layer architecture and integrity are significantly maintained in the final products, interfacial diffusion between neighboring layers is by-no-means negligible, even for completely immiscible polymers. Instead, interdiffusion driven by entropic advantage still occurs during coextrusion and the formed interphase between neighboring layers can strongly affect the properties of the multilayered polymers, especially for nanolayered structures. For example, a merging and even convergence of glass transition temperatures $\left(T_{g} \mathrm{~s}\right)$ with increasing number of layers (or decreasing layer thickness) was ever reported in multilayer films such as PC/PMMA systems, especially when the layer thickness was reduced below $10 \mathrm{~nm} .{ }^{12,18}$ The surprising merging of $T_{g} S$ similar to that commonly observed in miscible blends is due to a finite diffuse interphase of mixed component chains, indicating that interphase fraction becomes significantly higher and dominates the overall systems. Particularly, the multilayer films alternating of incompatible polymers even becomes totally interphase materials, ${ }^{18,19}$ when the layer thicknesses are close to or less than the theoretical interphase thickness $(5-10 \mathrm{~nm})$ by Helfand and co-worker's prediction. ${ }^{20}$ Similarly, other recent reports also revealed the dominant role of diffuse interphase from interdiffusion in the glass transitions for immiscible nanolayered systems. ${ }^{21,22}$ Apart from the effects on glass transition, the diffuse interphase in the immiscible nanolayered films also has a substantial effect on the gas permeability and 
mechanical properties. ${ }^{12,18,19}$ Therefore, it is understandable that the interphase from interfacial diffusion plays a crucial role in multilayered polymers. It is thus highly important to have a deep understanding of interdiffusion phenomenon and interphases development towards designing multilayered polymers with tailored performances.

Interdiffusion and interphase formation in multilayered structures can be better understood by coextruding alternating layers of two miscible polymers. Occasional articles reported the interdiffusion occurring in the coextrusion process. In a pioneering work, Baer and co-workers studied the interdiffusion in microlayered polymer systems such as PC/copolyesters, ${ }^{23}$ nylon/ethylene vinyl alcohol, ${ }^{24}$ and high density polyethylene/linear low density polyethylene. ${ }^{25}$ They proposed an interdiffusion model based on Fick's diffusion law that related the mutual diffusion coefficient to the change in the glass transition temperatures and crystallization behaviors. It is imperative to mention that the interdiffusion model proposed by Baer's group follows the slow-mode diffusion mechanism and assumes the symmetry of interdiffusion profile and that the mutual diffusion coefficient is independent of the composition. Very recently, Lamnawar and co-workers proposed a modified rheological model from a primitive Qiu-Bousmina model ${ }^{26}$ to determine the mutual diffusion coefficient, and subsequently mapped the interfacial composition profile in coextruded layers, on the basis of polymer dynamics theory and fast-controlled mode theory. ${ }^{27-29}$ It is worth mentioning that the composition profile determined by their rheology model agrees well with the one determined by energy dispersive X-ray (EDX) analysis. Alhough the interfacial diffusion has been studied and relevant models have been established, a clear understanding of its role in coextruded micro-/nanolayered structures is still lacking in the open literature. Besides, laminar flow conditions in the coextrusion combine polymers within the layer multipliers by producing large interfacial area without complete mixing. We can thus imagine that the diffuse interphases can prevail in the layered structures, strongly dependent on the contact time, the multipliers and layer thicknesses. The presence of such diffuse interphases might also largely alter the morphology and structure of the obtained multilayered polymers, as well as the resulting properties. Despite a large body of interesting work performed on multilayer coextrusion, few studies are available in the open reports addressing these effects from micro- to nanoscale. The measurement on the interfacial properties is pivotal for establishing the interdependence of processing, structure, and properties.

Therefore, in this work, we perform a systematical investigation of interfacial diffusion and diffuse interphase development in the multilayered polymer films produced by micro-/nanolayer coextrusion. Different from the frequently reported studies dealing with immiscible or partially miscible multilayer systems, our study is focused on a PVDF/PMMA pair that is completely miscible in the melt state. ${ }^{30}$ Using such a melt miscible pair as a model is advantageous, as it can amplify the contribution of interfacial diffusion present in the obtained multilayer structures. The objective of this study is to reveal the effects of interfacial diffusion and the formed diffuse interphases on morphology/microstructure 
development, dielectric properties and rheological behaviors of multi micro-/nanolayered films. We further attempt to understand the presence of diffuse interphases and map their length scales in these multilayer structures. The relationship of coextrusion process, structure, and properties will be emphasized. This work will offer some new enlightenment for understanding and tailoring the interfaces/interphases and structure in micro-/nanolayered polymer films towards the target properties.

\section{Experimental section}

\subsection{Materials and sample preparation}

PMMA (Altuglas V825T) and PVDF (Kynar 720) in granular pellet form were kindly provided by Arkema. Main characteristics of the polymers are summarized in Table 1. The characteristics of these polymers have been carefully investigated and described in our previous studies. ${ }^{27}$ To avoid water uptake, all the pristine polymers were dried in a vacuum oven at $80^{\circ} \mathrm{C}$ for $48 \mathrm{~h}$ prior to processing.

Table 1. Characteristics of the investigated polymers

\begin{tabular}{|c|c|c|c|c|c|c|c|c|c|}
\hline Polymer & $M_{\mathrm{w}}(\mathrm{kg} / \mathrm{mol})^{a}$ & PDI a & $T_{\mathrm{g}}\left({ }^{\circ} \mathrm{C}\right)^{b}$ & $T_{\mathrm{m}}\left({ }^{\circ} \mathrm{C}\right)^{b}$ & $T_{\mathrm{c}}\left({ }^{\circ} \mathrm{C}\right)^{b}$ & $\rho\left(\mathrm{g} / \mathrm{cm}^{3}\right)$ & $b(\AA)^{c}$ & $R_{\mathrm{g}}(\mathrm{nm})^{d}$ & $\begin{array}{l}\text { Reptation } \\
\text { time, } \tau_{\text {rep }} \\
\left(\mathrm{s}, 220^{\circ} \mathrm{C}\right)\end{array}$ \\
\hline PVDF & 210 & 2.0 & -42 & 170 & 136 & 1.78 & 10.6 & 11.98 & 0.37 \\
\hline PMMA & 100 & 1.9 & 112 & - & - & 1.19 & 15.5 & 8.41 & 0.56 \\
\hline
\end{tabular}

a Weight-average molecular weight $\left(M_{w}\right)$ and polydispersity index (PDI) were determined by size exclusion chromatography with tetrahydrofuran as the eluent for PMMA and dimethyl formamide for PVDF.

${ }^{b}$ Glass transition temperature $\left(T_{\mathrm{g}}\right)$, melting temperature $\left(T_{\mathrm{m}}\right)$ and crystallization temperature $\left(T_{\mathrm{c}}\right)$ were measured using the differential scanning calorimetry (DSC, Q20, TA Instruments) at a heating/cooling rate of $10^{\circ} \mathrm{C} / \mathrm{min}$ under $\mathrm{N}_{2}$.

${ }^{c}$ The effective bond length $(b)$ (Kuhn segment length) was calculated by $b=C_{\infty} l / \sin \left(109.5^{\circ} / 2\right)$ with the characteristic ratio $\left(C_{\infty}\right) 5.6$ for PVDF and 8.2 for PMMA, C-C bond length $l=1.54 \AA$, and the bond angle of $109.5^{\circ}$ between $\mathrm{C}-\mathrm{C}$ bonds in the backbone. ${ }^{31}$

${ }^{d}$ The radius of gyration $\left(R_{g}\right)$ was determined by $\left\langle R_{g}{ }^{2}\right\rangle=\left\langle R^{2}\right\rangle / 6$, with the ratio of mean-square end-to-end distance $\left\langle R^{2}\right\rangle$ to molar mass $M\left(<R^{2}>/ M\right) 0.41 \AA^{2} \mathrm{~mol} / \mathrm{g}$ for PVDF and $0.425 \AA^{2} \mathrm{~mol} / \mathrm{g}$ for PMMA. ${ }^{32}$

PVDF/PMMA multilayer films were produced using a forced-assembly multilayer coextrusion process. As schematically shown by Figure 1, the coextrusion system is composed of two single-screw extruders (A and B), a coextrusion feedblock, a set of vertical layer multipliers, an exit flat die, and a thermally regulated chill roll. The multiplier employed here has a constant cross-sectional area and ensures a balanced and symmetrical flow. Different from the compression-then-expansion step of the standard multiplier, this new-generation multiplier simultaneously compresses the materials in one direction and expands in another, which is favorable for minimizing the flow nonuniformities with rheological mismatched materials. From the feedblock where two melt streams combine (Figure 1), the initial two 
layers flow through a sequence of multipliers. The melt is initially sliced vertically, and then the halves are spread horizontally to the original width and finally recombined, while keeping the total thickness of the melt constant, thus doubling the number of layers and reducing the thickness of each layer by a factor of 2 after each multiplier. The number of layers $(N)$ in as-coextruded films is determined by the number of multiplier elements $(n)$ as $N=2^{\mathrm{n}+1}$ (Figure 1).

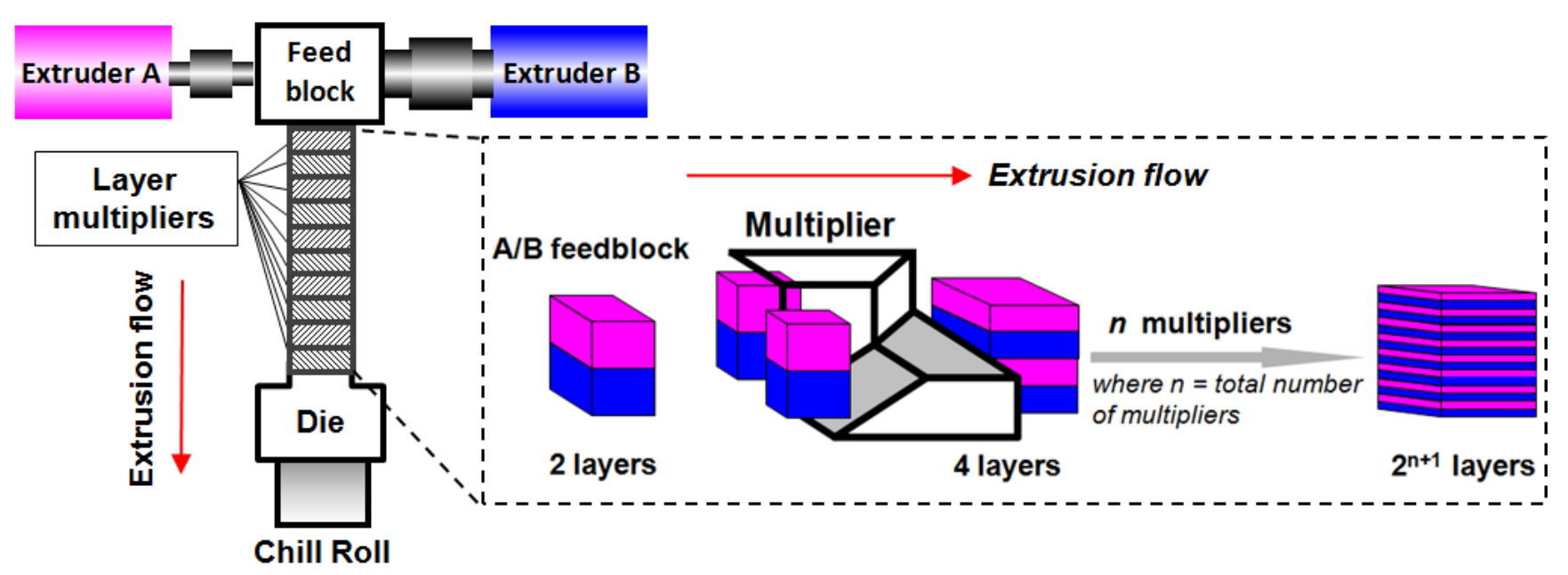

Figure 1. Schematic of the layer multiplication in the two-component (A/B) multilayer coextruder employed in this study.

Table 2. Characteristics of the multilayered PVDF/PMMA films

\begin{tabular}{lcccc}
\hline \multirow{2}{*}{ Samples } & no. of & no. of & \multicolumn{2}{c}{ Nominal layer thickness } \\
\cline { 4 - 5 } multipliers $(n)$ & layers $(N)$ & 2 & $104 \mu \mathrm{m}$ & PMMA \\
\hline 2L & 0 & 8 & $26 \mu \mathrm{m}$ & $116 \mu \mathrm{m}$ \\
$8 \mathrm{~L}$ & 2 & 32 & $6.5 \mu \mathrm{m}$ & $29 \mu \mathrm{m}$ \\
$32 \mathrm{~L}$ & 4 & 256 & $813 \mathrm{~nm}$ & $7.25 \mu \mathrm{m}$ \\
$256 \mathrm{~L}$ & 7 & 2048 & $102 \mathrm{~nm}$ & $906 \mathrm{~nm}$ \\
$2048 \mathrm{~L}$ & 10 & & & $113 \mathrm{~nm}$ \\
\hline
\end{tabular}

Here, 0, 2, 4, 7 and 10 multipliers were employed, thus giving the fabricated films containing 2, 8, 32, 256 and 2048 layers, respectively (Table 2). The nomenclature for the multilayer films is " $N L$ ”, where $N$ is the number of layers. The extruder, multiplier elements and die temperatures were set to $220^{\circ} \mathrm{C}$ to have matching viscosities of the two polymer melts in the shear-rate range of the coextrusion process, typically between 1 and $10 \mathrm{~s}^{-1}$ (see Figure S1 in the Supporting Information), and also to ensure the thermostability of polymers during processing. After the die exit, the layered melt quenched onto a chill roll to form a film. In this study, equal volumetric flow rates were used to prepare the model multilayer film of PVDF/PMMA with the component volume ratio of 50/50. The chill roll was set to $80{ }^{\circ} \mathrm{C}$, and a negligibly low drawing speed was used to allow for a reduction of the total film thickness without stretching. Films 
were produced in a continuous manner with approximately $220 \mu \mathrm{m}$ total thickness. The nominal layer thicknesses for those as-coextruded multilayer PVDF/PMMA (50/50) films are calculated according to:

$$
h_{\text {nom }, A}=\frac{\phi_{A}}{2^{n}} h_{\text {total }}
$$

where $\phi_{A}$ is the volume fraction of solid-state polymer $A$ and $h_{\text {total }}$ is the total thickness of the multilayer film. This equation works similarly for polymer B. The individual layer thicknesses were further corrected by the density changes for polymers from the melt to solid state and reported in Table 2. The layer thicknesses were further checked by the morphological investigations. Besides, control films of neat polymers were also extruded under the same conditions for the sake of comparison. Additionally, a PVDF/PMMA blend with a volume composition of 50/50 was also prepared by melt blending at $220{ }^{\circ} \mathrm{C}$ using a twin-screw extruder. ${ }^{33}$

\subsection{Characterizations}

Polarized optical microscopy. A polarized optical microscope (Axio Scope.A1, Zeiss) was used to visualize the multilayer structures and their integrity. A thin slice about $15 \mu \mathrm{m}$ in thickness was cut from an extrudate using a cryo-ultramicrotome at $-80{ }^{\circ} \mathrm{C}$ with a diamond knife blade. Morphological pictures of the cross-sections were taken by a CCD camera connected to the microscope.

Atomic force microscopy (AFM). Atomic force microscopy (AFM, Multimode 8, Bruker) was employed to observe the multilayer structure in multilayer films. Measurements were carried out under ambient conditions at room temperature. Tapping mode was performed at a scan rate of $0.5 \mathrm{~Hz}$ with uncoated silicon probes. A flat and smooth cross-section of the film was obtained from cryo-ultramicrotoming at $-80{ }^{\circ} \mathrm{C}$. Layer continuity and uniformity of the films were characterized. Phase and height images of the cross-sections were recorded.

Transmission electron microscopy (TEM). A transmission electron microscope (Philips CM120) was employed to observe the cross-sectional morphology of the multilayer films. Thin samples for TEM analysis were cut perpendicular to the extrusion direction (ED) using the cryo-ultramicrotome.

Scanning electron microscopy (SEM) and Energy dispersive $X$-ray (EDX) analysis. Scanning electron microscope (SEM, S800 FEG, Hitachi) in combination with energy dispersive X-ray (EDX) analysis was conducted across interfaces of the multilayer films to determine the component concentration profile across the layer thickness. This technique makes use of the characteristic X-ray fluorescence of characteristic atoms in the sample, allowing a direct measurement of the concentration gradient of components in the interfacial region. ${ }^{34}{ }^{35}$ In the PVDF/PMMA systems under investigation, a unique fluorine (F) element of PVDF and oxygen (O) element of PMMA provide good markers for PVDF and PMMA, respectively. By probing the traces of the atomic $\mathrm{F}$ and $\mathrm{O}$ compositions across the interfacial region, we could obtain the concentration profiles of PMMA and PVDF versus their positions. Multilayer specimens were first microtomed normal to the interfacial plane using an ultramicrotome. A line scan of 
electron beam was conducted on the cross-sections from one layer to the others. The EDX analyzer collected the X-ray data across this path, and at each point the number of X-ray events of given energy generated was recorded as a function of X-ray energy. ${ }^{27}$

$\boldsymbol{X}$-ray characterizations. The crystalline structure and morphology of multilayer films were studied with two-dimensional (2D) wide- and small-angle X-ray scattering (2D-WAXS and SAXS) measurements performed on the BM02-D2AM beamline of the European Synchrotron Radiation Facility (Grenoble, France). The as-coextruded multilayer film was placed on a home-made sample holder (without stacking films), with the film extrusion direction parallel to the incident beam. The incident photon energy was set to $17 \mathrm{keV}$, and the beam diameter was in the order of $100 \mu \mathrm{m}$. The sample-to-detector distance was $11.0 \mathrm{~cm}$ for WAXS and $1.63 \mathrm{~m}$ for SAXS. The scattering vector $q(q=$ $4 \pi \sin \theta / \lambda$, where $\theta$ is the half of the scattering angle and $\lambda$ is the incident wavelength) was calibrated using a silver behenate standard for SAXS and lanthanum hexaboride $\left(\mathrm{LaB}_{6}\right)$ for WAXS. The data acquisition time for each pattern was $20 \mathrm{~s}$. Two-dimensional data were corrected by considering the camera distortion, dark image reading and flat field response of the detector. The scattering intensity was further normalized by the incident flux, the sample thickness, and intensity-calibration was realized using a glassy carbon standard.

Differential scanning calorimetry (DSC). Thermal properties were explored by a differential scanning calorimeter (DSC, Q20, TA Instruments) with a dry nitrogen purge. Approximately $5 \mathrm{mg}$ of sample were loaded into the DSC aluminum pan. A temperature program was prescribed in which the samples were first heated from -90 to $220{ }^{\circ} \mathrm{C}$, and then cooled to $-90{ }^{\circ} \mathrm{C}$ followed by a second heating scan to $220{ }^{\circ} \mathrm{C}$. Both the heating and cooling rates were $10^{\circ} \mathrm{C} / \mathrm{min}$.

Dielectric relaxation spectroscopy (DRS). Dielectric relaxation spectroscopy (DRS) measurements were conducted on a precision LCR meter (Agilent E4980A) equipped with the Environmental Test Chamber (ETC, TA Instruments) and Air Chiller System (ACS-3, TA Instruments) for temperature control. A sinusoidal electric field (2 V amplitude) was applied in a frequency range between $20 \mathrm{~Hz}$ and $2 \mathrm{MHz}$ to determine the complex permittivity. Disks were punched from the as-coextruded films and positioned between two parallel-plate brass electrodes (25 mm in diameter). A first heating until a temperature $T_{0}\left(T_{\mathrm{c}}<\right.$ $T_{0}<T_{\mathrm{m}}$ ) was performed to ensure a good thermal contact between the electrodes and the film. Data were recorded during the cooling to $-70^{\circ} \mathrm{C}$ at a rate of $5^{\circ} \mathrm{C} / \mathrm{min}$ and upon the second heating up to several high temperatures to check the data reproducibility. Besides, dielectric responses were also recorded isothermally with fresh samples at a temperature of $220^{\circ} \mathrm{C}$. Before starting tests, the sample was heated at the experimental temperature for $60 \mathrm{~s}$ in the ETC oven to pre-melt the sample and also to ensure a good contact between sample and electrodes. The data collection period for a frequency sweep from $20 \mathrm{~Hz}$ to 2 $\mathrm{MHz}$ is $\sim 11 \mathrm{~s}$. Nitrogen purging at a flow rate of $10 \mathrm{~L} / \mathrm{min}$ was maintained throughout the tests. All measurements were repeated at least three times with fresh samples to check the reproducibility. 
Shear rheology. (1) Dynamic frequency sweep. Linear viscoelasticity of neat polymers was obtained from small-amplitude oscillatory shear (SAOS) flow using a rotational Discovery Hybrid Rheometer (DHR-2, TA Instruments) with a parallel-plate geometry (25 mm in diameter) at temperatures from 180 to $240{ }^{\circ} \mathrm{C}$. Dried pristine polymer pellets were compression-molded into disks at $220^{\circ} \mathrm{C}$ with a pressure of 200 bar, and further dried at $80{ }^{\circ} \mathrm{C}$ in a vacuum oven for $24 \mathrm{~h}$ before measurements. Disks with the thickness of $c a .1200 \mu \mathrm{m}$ were placed between the plates, and left for $5 \mathrm{~min}$ at the measured temperature to melt/relax the polymer before tests. Measurements were performed under a fixed strain amplitude $\left(\gamma_{0}\right)$ of $5 \%$, which lies in the linear viscoelastic (LVE) regime, from the angular frequency $(\omega)$ of 628 to $0.01 \mathrm{rad} / \mathrm{s}$. For all rheological tests, experiments were conducted under nitrogen purging in an enclosed oven. Linear viscoelasticity of studied polymers is reported in Figure S1 in the Supporting Information.

(2) Dynamic time sweep. The thermal stability of neat polymers used was examined beforehand by a dynamic time sweep test for $50 \mathrm{~min}$ at a temperature of $220^{\circ} \mathrm{C}$, strain amplitude $\left(\gamma_{0}\right)$ of $1 \%$, and angular frequency of $1.0 \mathrm{rad} / \mathrm{s}$ under nitrogen (see Figure S2). The interfacial diffusion process of the PVDF/PMMA bilayer in the melt was tracked using SAOS measurement to decouple the flow effects. Prior to measurement, PVDF and PMMA disks with individual thicknesses of $600 \mu \mathrm{m}$ were respectively sticked to the upper and lower plates of the rheometer at the studied temperature $220{ }^{\circ} \mathrm{C}$, and subsequently kept for 3 min to erase the residual stress and improve the adhesion between samples and plates. Then, the upper geometry with PVDF layer was lowered to become in close contact with the PMMA layer on the lower plate. Dynamic time sweep tests were then performed to probe the healing process of the bilayer at $220^{\circ} \mathrm{C}$ under nitrogen with a frequency of $1.0 \mathrm{rad} / \mathrm{s}$ and strain of $1.0 \%$. Here, time zero was defined as the time when the bilayer was in intimate contact. Monolayers (neat polymers) were also tested by the same procedure for reference.

Uniaxial extensional rheology. Uniaxial extensional rheology was carried out using a Sentamanat Extensional Rheometer fixture (SER-2, Xpansion Instruments, LLC) mounted on the DHR-2 rheometer. ${ }^{29 \text {, }}$ ${ }^{36}$ Samples from the extrusion process were cut into rectangular strips with the following dimensions: length, width and thickness of $20 \mathrm{~mm} \times 10 \mathrm{~mm} \times 0.2 \mathrm{~mm}$ (length along the extrusion direction) for the extensional test. Each sample was pre-heated at the tested temperature for $120 \mathrm{~s}$ in the rheometer oven before tests, and was observed with a built-in camera to ensure sagging did not occur. Extensional flow data were then collected with extension parallel to the film extrusion direction at a constant Hencky strain rate at $220^{\circ} \mathrm{C}$ under nitrogen. All measurements were repeated at least three times.

\section{Results and discussion}

\subsection{Morphology and microstructure analyses}

Figure $2 \mathrm{a}-\mathrm{c}$ illustrates the morphology features captured under the optical microscopy for the as-coextruded PVDF/PMMA multilayer films varying number of layers. Generally, those coextruded 
films show a continuous multilayered architecture, with no clear evidence of interfacial instability. The remarkable interfacial stability is attributed to the miscibility of components. Discrete layers of PVDF and PMMA are clearly identified especially at a microlayered film (e.g., 8L), where the rough textured layers are semi-crystalline PVDF and the smooth layers are the amorphous PMMA (Figure 2a). With a smaller number of layers, the film shows some fluctuations in the individual layer thicknesses (Figure 2a), which is possibly caused by the multiplication process during coextrusion, as well as viscoelastic differences between two polymers (Figure S1). Nevertheless, the layer thickness uniformity is improved by increasing the number of layers (Figure 2b, c), which is confirmed by the AFM image for 256L film (Figure 2d). This is because of the reduced contrast in viscoelasticity between components by the enhanced interlayer diffusion at the longer layer-layer contact. Therein, the diffuse interphases comprising a graded mixture of PVDF/PMMA are increasingly created by increasing the number of layers, which act as bridges between neighboring layers. Also, an increased homogeneity in the dissipation of multilayered flows with the larger number of layers should also favor the layer uniformity. Besides, we can also observe that the interfaces among layers are clearly sharp for a microlayered film of 8L. This is due to the fact that when the polymers are just combined, there is negligible interlayer diffusion. It is imperative to mention that the PVDF/PMMA pair is completely miscible in the melt. One might thus expect that interdiffusion will greatly occur in films with a larger number of layers considering the longer melt residence within coextrusion channels, and layer morphology should be indiscernible. However, it is interesting to note that the layer morphology is still visible for films containing a greater number of layers, and the films are not yet homogeneous as miscible blends. As demonstrated by both optical and AFM micrographs (Figure 2c,e), the 2048L film still displays the multilayered morphology, where the discrete PVDF layers (dark) and PMMA layers (bright) are noticeable in the AFM phase images. Note that the dark region present on the right of Figure 2e is PVDF crystals covering several layers, because it is close to the earlier formed diffuse interphase regions where interdiffusion lasted for a longer time (the evolution of interphases will be explained later). Multilayered morphology in 2048L is further verified by TEM observation in the following. That is to say, continuous layer structure could be maintained in nanolayered films despite the substantial interdiffusion among layers, making them distinguishable from homogeneous blends. 

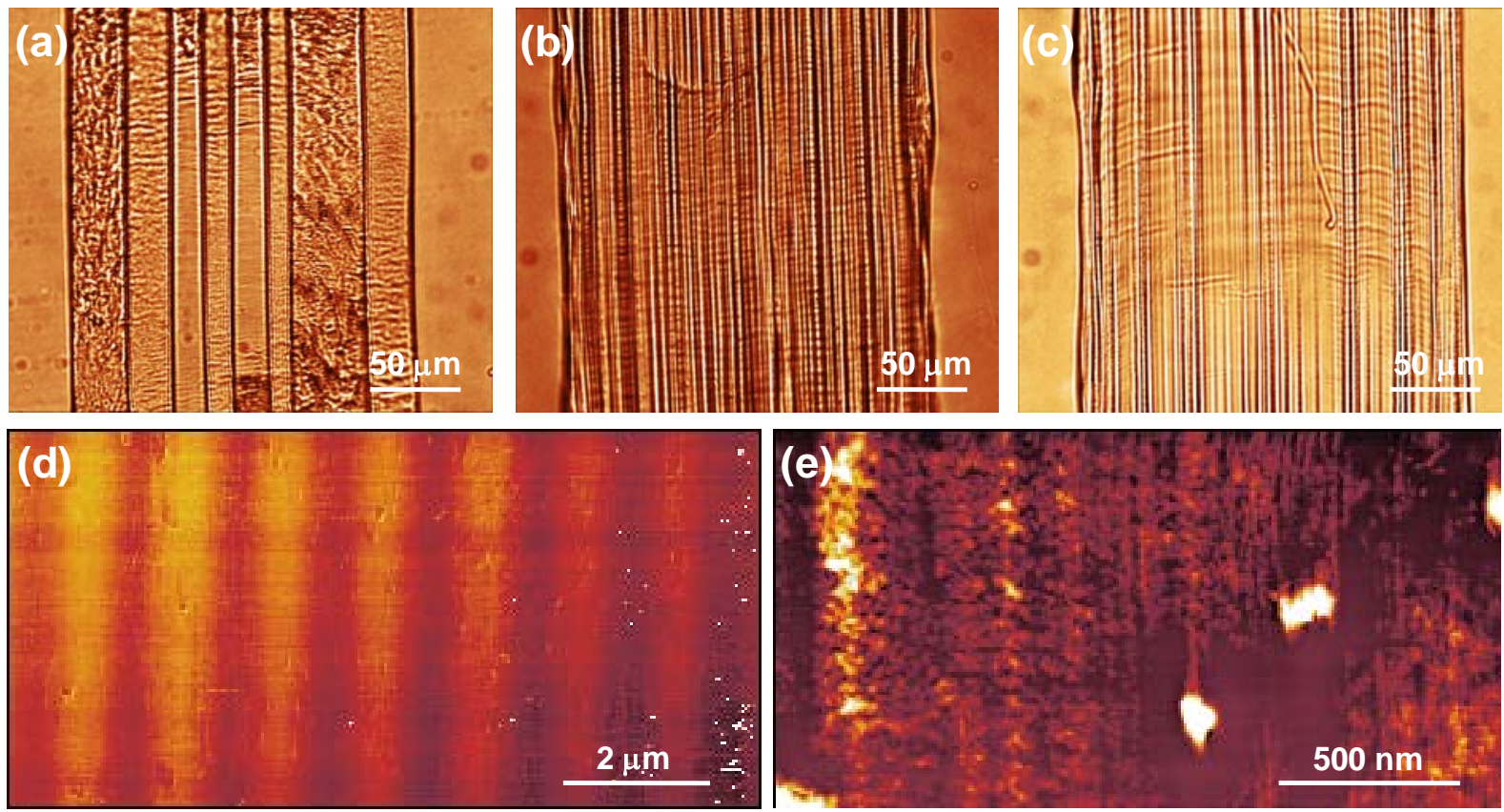

Figure 2. Optical micrographs of cross-sections for PVDF/PMMA multilayer films with various number of layers: (a) 8L, (b) 256L and (c) 2048L. AFM phase images of the cross-sections for (d) 256L, and (e) 2048L. PVDF layers appear dark and PMMA layers appear bright in the AFM images.

The crystalline structure and morphology of PVDF in those multilayer films were investigated by simultaneous WAXS/SAXS techniques. Figure 3a-h representatively shows the 2D-WAXS and SAXS patterns recorded for the multilayer films with X-ray incident beam along the extrusion direction (ED). The corresponding one-dimensional (1D) curves integrated azimuthally from 2D patterns are plotted in Figure $3 \mathrm{i}-\mathrm{j}$. In all multilayer films prepared, PVDF crystallized typically in the form of $\alpha$-phase, as observed from the intense diffraction rings/peaks indexed as: (100), (020), (110) and (021) planes (Figure 3a-h,i). Besides, from the WAXS/SAXS patterns we can notice the oriented crystallization along the extrusion direction in these films, despite the negligible draw-down ratios for film collection. The equatorial (100) and (020) reflections in WAXS patterns indicate the presence of an edge-on lamellar orientation with the $b$-axis perpendicular to the layers (Figure 3a-c), especially for 32L and 256L films. For the 8L film, the weaker crystal and lamellar orientations are observed from the nearly random WAXS reflections and meridian arc-like SAXS scattering. Note that the strong streak present along the equator of SAXS patterns for multilayer films also appears in the neat PVDF and PMMA control films (Figure S3) and has been ever observed in other multilayer systems. ${ }^{14,37}$ This is mainly due to the grazing incidence scattering from surface, interface, etc., ${ }^{37,38}$ and thus not the focus of the present study. For the 32L and 256L films, both PVDF crystals and lamellae show the stronger orientation along ED, as evidenced by the more intense equatorial reflections in WAXS patterns and the meridian tear-drop-like SAXS patterns. Considering the fact that all films were taken up by the chill roll from the extrusion die at the same speed (i.e., total film thicknesses are the same as ca. $220 \mu \mathrm{m}$ ), the improved orientations in the 32L film 
compared to that of the 8L film should originate from the layer confinement via the additional multiplication process (i.e., 4 multipliers for 32L versus 2 multipliers for 8L). For films containing more layers, the orientations are slightly decreased, as observed for 2048L (Figure 3d,h). It also has to be noted that more dispersed diffraction rings with reduced intensity are observed with the increase in the number of layers (Figure 3a-c), suggesting that crystallization of PVDF is decreased in those multilayer films. The suppressed crystallization by increasing the number of layers is also viewed from DSC results (Table 3, Figure S4), which is mainly because of the interdiffusion between PMMA and PVDF segments during coextrusion. Coextrusion through an increasing number of multipliers increases the number of interfaces and the melt contact time between adjacent layers whereas it decreases the layer thickness. Consequently, the amount of interdiffusion remarkably increases and forces the entire melt towards a homogeneous blend after several multiplications. The decreased crystallization by increasing the content of PMMA in PVDF/PMMA blends has been widely accepted ${ }^{39,40}$ and was also observed in our recent work, ${ }^{41}$ which is attributed to the macromolecular confinement on the entanglement spacing and/or chain surroundings of PVDF imposed by PMMA segments. Similar to the blending, the inter-mixing between PMMA and PVDF at their interface of multilayer systems causes confinement on the movement of PVDF chains, thus suppressing its nucleation and subsequent crystallization. The more interdiffusion between the two polymers, the lower crystallinity $\left(X_{c}\right)$ and the smaller lamellar thickness $\left(L_{c}\right)$ can be thus observed for multilayer films (Table 3). Besides, the long period $L_{w}\left(L_{w}=2 \pi / q_{\max }\right)$ of crystals becomes larger with the increase in the number of layers (Figure $3 \mathrm{j}$ and Table 3). This is due to the inclusion of PMMA in the interlamellar spaces of PVDF by interdiffusion, which increases the periodic spacing of crystals. ${ }^{42}$ Moreover, it is also evident that the 2048L film exhibits lower crystallinity and thinner lamellae than the equivalent PVDF/PMMA (50/50) blend (Figure 3i-j and Table 3). This suggests that apart from the macromolecular confinement from rigid PMMA segments via interdiffusion, the geometrical confinement from the layer multiplication further suppresses the PVDF crystallization in multilayer films. This is because of the fact that, different from blends, the PVDF layers in multilayer films crystallize within the space between two neighboring PMMA layers that are hard and amorphous. Layer multiplication gradually reduces the individual layer thicknesses, when it increases the total number of layers. Logically, in the nanolayered 2048L film, those spaces allowing for crystallization are greatly reduced by the layer multiplication; thus, the crystal growth is limited in those highly confined spaces. ${ }^{9}$ This concern will be further verified by the following TEM investigations. 

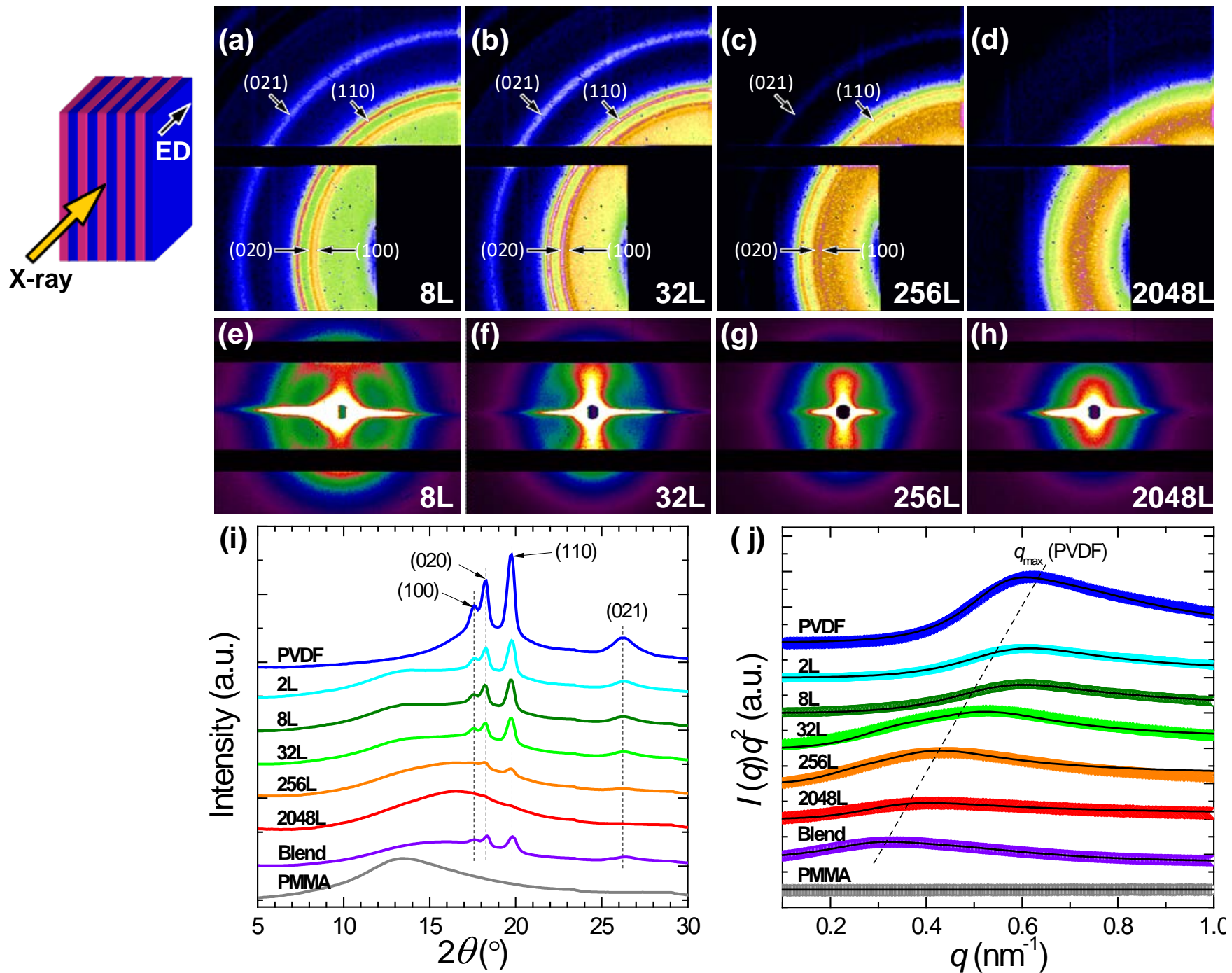

Figure 3. Two-dimensional (2D)-WAXS (a-d) and SAXS (e-h) patterns recorded with X-ray beam parallel to the extrusion direction (ED) for PVDF/PMMA multilayer films: (a,e) 8L, (b,f) 32L, (c,g) 256L and (d,h) 2048L. The scheme appended at the left side shows the configuration of the X-ray beam and film during tests. (i) One-dimensional (1D)-WAXS profiles and (j) Lorentz-corrected 1D-SAXS profiles for films. Solid curves in (j) are fits to the Lorentzian function. Curves in (i) and (j) are shifted vertically for clarity. The intensity was normalized with film thicknesses.

Interfacial morphology of multilayer films was further examined using TEM. Figure 4 depicts the representative TEM micrographs for cross-sections in the microlayered 8L film, and nanolayered 2048L. We can notice in the microlayered 8L film (Figure 4a-c) that the interfacial region between PVDF and PMMA is clearly sharp and there is a negligibly thinner diffuse interphase formed in between (Figure 4c). Evidently, PVDF-rich regions far from the interface display the typical spherulite morphology with little effect of confinements. However, PVDF lamellae nearest to the interface show the weak edge-on orientation (see arrows in Figure 4b,c), resulting from the layer multiplication. For the nanolayered 2048L film (Figure 4d-f), we observe the wider distribution of PVDF crystals across the layers due to the substantial interdiffusion, especially for the regions close to the earlier formed diffuse interphases where the interdiffusion lasted for a longer contact time (Figure 4d,e). Note that the presence of PVDF- or 
PMMA-rich areas covering several hundred nanometers in those regions is due to the interdiffusion at the longer contact during coextrusion, as well as the crystallization induced phase separation during cooling. ${ }^{42}$ Nevertheless, we can still distinguish the PVDF-rich layers (dark) and PMMA-rich layers (bright) (see the areas sandwiched between parallel lines in Figure 4e,f). Herein, individual thicknesses of PMMA layers in 2048L are around $100 \mathrm{~nm}$ or less, which are slightly less than the expected nominal thicknesses because of the reduction in layer thickness by interlayer diffusion.
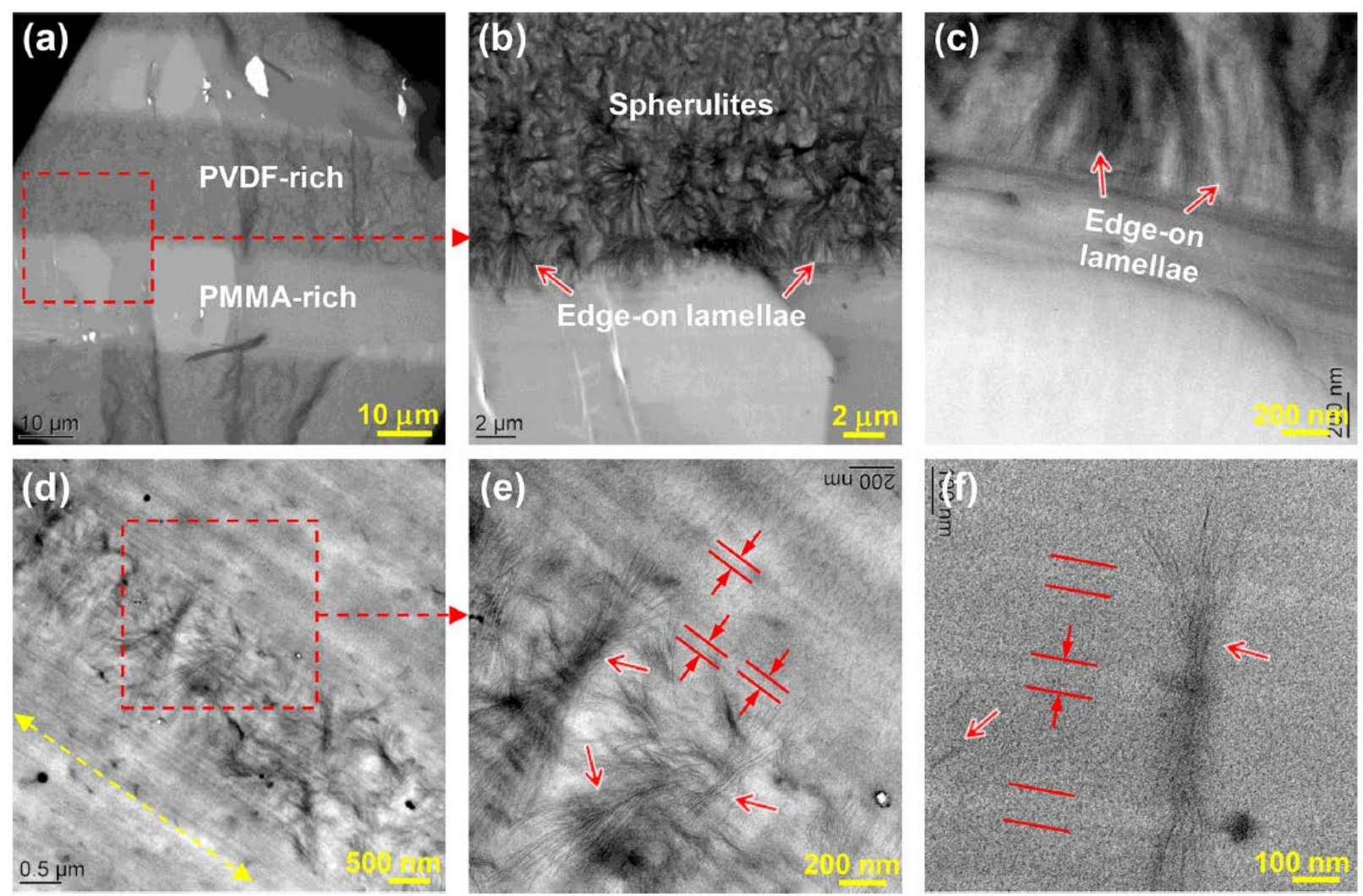

Figure 4. TEM micrographs showing the cross-sections of PVDF/PMMA multilayer films: (a-c) 8L and (d-f) 2048L. (b, c) Enlarged images of (a) at the interfacial regions for 8L and (e, f) enlarged images of (d) for 2048L. Dark layers are PVDF-rich regions and bright layers are PMMA-rich regions. Double-headed arrow in (d) indicates the film in-plane direction, and single arrows in (e) and (f) show the edge-on lamellae. The area sandwiched between parallel lines in (e) and (f) indicates the discrete layers.

Besides, the distance between two neighboring PMMA layers could be as large as $200 \mathrm{~nm}$ (Figure 4f), because it includes two diffuse interphases in addition to a PVDF-rich layer. It is also worth noting that compared with the 8L film, PVDF crystals in the 2048L are smaller and coarser, and mainly bundle-like crystals with stacked edge-on lamellae oriented normal to the layer surface (see arrows in Figure 4e,f). Obviously, crystals in the 2048L film are less perfect as compared with typically spherulitic morphology in the PVDF/PMMA (50/50) blend. ${ }^{43}$ Herein, macromolecular confinement from PMMA segments in the 2048L film is insufficient to cause such imperfect crystals with the lower crystallinity. This is because 
that the degree of PMMA confinement in a multilayered structure should be lower than that in the blend because of the incomplete mixing compared with that in the latter. Instead, the spatial confinement from layer multiplication thus further reduces the crystallization, considering that the layer thicknesses are reduced to only 10 times the radius of gyration of PVDF (Table 1 ). ${ }^{9}$ This finding agrees well with the $\mathrm{X}$-ray and DSC results above.

Table 3. Melting temperature $\left(T_{\mathrm{m}}\right)$, enthalpy of fusion $\left(\Delta H_{m}\right)$, crystallinity $\left(X_{\mathrm{c}}\right)$ and PVDF lamellar thickness $\left(L_{\mathrm{c}}\right)$ determined from the first heating scan of DSC, and long period $\left(L_{\mathrm{w}}\right)$ calculated by SAXS

\begin{tabular}{cccccc}
\hline Samples & $T_{m}\left({ }^{\circ} \mathrm{C}\right)$ & $\Delta H_{m}(\mathrm{~J} / \mathrm{g})$ & $X_{\mathrm{c}}(\%)$ & $L_{c}(\mathrm{~nm})$ & $L_{w}(\mathrm{~nm})$ \\
\hline PVDF & 169.4 & 54.74 & 52.3 & 8.64 & 10.16 \\
2L & 169.3 & 27.10 & 51.8 & 8.63 & 10.21 \\
8L & 167.8 & 26.60 & 50.8 & 8.29 & 10.34 \\
32L & 166.8 & 21.45 & 41.0 & 8.12 & 11.82 \\
256L & 159.3 & 17.57 & 33.6 & 6.91 & 14.70 \\
2048L & 155.6 & 15.67 & 29.9 & 6.44 & 15.62 \\
Blend (50/50) & 159.5 & 17.33 & 33.1 & 6.98 & 13.79 \\
\hline
\end{tabular}

\subsection{Dielectric properties of coextruded multilayer films}

Figure 5a-c compares the dielectric spectra as a function of frequency for as-coextruded PVDF/PMMA multilayer films at a temperature of $30^{\circ} \mathrm{C}$, as well as those for control films. The corresponding dielectric spectra against temperature at the tested frequency of $1 \mathrm{kHz}$ are plotted in Figure $5 \mathrm{~d}-\mathrm{f}$. For comparison, theoretical spectra of the multilayer system without any layer-layer interphases are calculated and also shown in Figure 5a-c (see dashed lines), according to the following equations: ${ }^{44,45}$

$$
\frac{1}{\varepsilon_{\mathrm{net}}^{*}}=\frac{\phi_{\mathrm{A}}}{\varepsilon_{A}^{*}}+\frac{\phi_{B}}{\varepsilon_{B}^{*}}
$$

where $\varepsilon_{\text {net }}^{*}=\varepsilon_{\text {net }}^{\prime}-i \varepsilon_{\text {net }}^{\prime \prime}$ represents the global complex permittivity function for the layered system, which is the same for $\varepsilon_{A}^{*}$ and $\varepsilon_{B}^{*}$ of constituent $\mathrm{A}$ and $\mathrm{B}$ layers; $\phi_{\mathrm{A}}$ is the volume fraction of layer A defined by the ratio of layer A thickness to the total thickness of the system (the same for layer B). Separating the real and imaginary parts of complex permittivity leads to:

$$
\begin{aligned}
& \varepsilon_{\text {net }}^{\prime}=\frac{\left[\left(\varepsilon_{A}^{\prime} \varepsilon_{B}^{\prime}-\varepsilon_{A}^{\prime \prime} \varepsilon_{B}^{\prime \prime}\right)\left(\phi_{B} \varepsilon_{A}^{\prime}+\phi_{A} \varepsilon_{B}^{\prime}\right)+\left(\varepsilon_{A}^{\prime} \varepsilon_{B}^{\prime \prime}+\varepsilon_{A}^{\prime \prime} \varepsilon_{B}^{\prime}\right)\left(\phi_{B} \varepsilon_{A}^{\prime \prime}+\phi_{A} \varepsilon_{B}^{\prime \prime}\right)\right]}{\left(\phi_{B} \varepsilon_{A}^{\prime}+\phi_{A} \varepsilon_{B}^{\prime}\right)^{2}+\left(\phi_{B} \varepsilon_{A}^{\prime \prime}+\phi_{A} \varepsilon_{B}^{\prime \prime}\right)^{2}} \\
& \varepsilon_{\text {net }}^{\prime \prime}=\frac{\left[\left(\varepsilon_{A}^{\prime} \varepsilon_{B}^{\prime \prime}+\varepsilon_{A}^{\prime \prime} \varepsilon_{B}^{\prime}\right)\left(\phi_{B} \varepsilon_{A}^{\prime}+\phi_{A} \varepsilon_{B}^{\prime}\right)-\left(\varepsilon_{A}^{\prime} \varepsilon_{B}^{\prime}-\varepsilon_{A}^{\prime \prime} \varepsilon_{B}^{\prime \prime}\right)\left(\phi_{B} \varepsilon_{A}^{\prime \prime}+\phi_{A} \varepsilon_{B}^{\prime \prime}\right)\right]}{\left(\phi_{B} \varepsilon_{A}^{\prime}+\phi_{A} \varepsilon_{B}^{\prime}\right)^{2}+\left(\phi_{B} \varepsilon_{A}^{\prime \prime}+\phi_{A} \varepsilon_{B}^{\prime \prime}\right)^{2}}
\end{aligned}
$$

These equations assume that no interphase layer is formed between neighboring layers, and any changes in the dielectric spectra of neat materials as a function of thickness are negligible. 


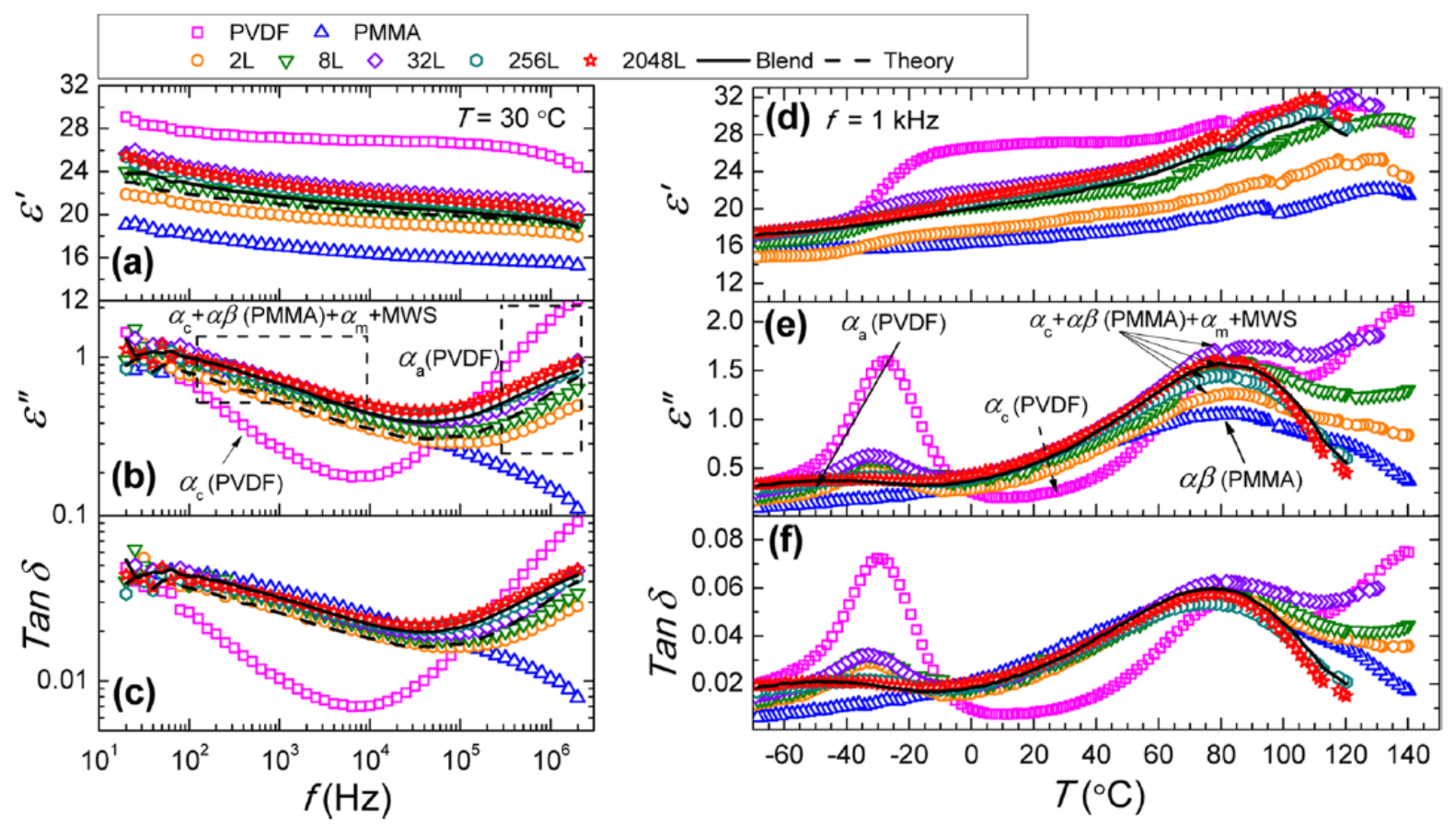

Figure 5. (a) Storage permittivity, (b) loss permittivity, and (c) loss tangent spectra versus frequency at $30{ }^{\circ} \mathrm{C}$ for multilayer and control films. (d-f) Corresponding dielectric spectra vs temperature at $1 \mathrm{kHz}$.

As well documented, for PVDF, the dielectric storage permittivity is mainly contributed by dipole polarizations of segments within the crystalline and/or amorphous regions. ${ }^{43}$ This generally manifests as one dielectric loss peak due to $\alpha_{\mathrm{c}}$-relaxation ("c" stands for "crystalline") at the lower frequencies (or higher temperatures), and the other assigned to $\alpha_{\mathrm{a}}$-relaxation ("a" for "amorphous") at the higher frequencies in the spectra shown in Figure 5b-c and e-f. However, dielectric constant in PMMA is resulting from dipole polarizations of segments and/or side groups, as reflected by $\alpha$-relaxation (segmental motions) and $\beta$-relaxation (local motions of side groups) (both relaxations always merge as $\alpha \beta$-relaxation) in Figure 5b-c and e-f. ${ }^{43}$ As observed in Figure 5a, the values of $\varepsilon^{\prime}$ at $30^{\circ} \mathrm{C}$ for multilayer films fall in between those of neat PVDF and PMMA. For all films, $\varepsilon^{\prime}$ is improved by increasing the temperature below the melting temperature (Figure $5 \mathrm{~d}$ ) because the dipoles become more activated upon heating. For multilayer films, various polarizations contribute to $\varepsilon^{\prime}$, involving dipole polarizations associated with PVDF crystals ( $\alpha_{\mathrm{c}}$-relaxations), PMMA $(\alpha \beta)$, miscible PVDF/PMMA regions $\left(\alpha_{\mathrm{m}}\right)$ and PVDF amorphous regions $\left(\alpha_{\mathrm{a}}\right.$ ) (see marks in Figure $5 \mathrm{~b}, \mathrm{e}$ ), ${ }^{43}$ as well as interfaces within the material. ${ }^{46}$

Obviously, experimental dielectric spectra at $30{ }^{\circ} \mathrm{C}$ for the $2 \mathrm{~L}$ film almost follow the theoretical predictions. Note that the theoretical spectra were extracted from the measured spectra of two components, assuming that no interphase layers are present between neighboring layers. Such an agreement between the experimental and theoretical values for the $2 \mathrm{~L}$ film indicates that a negligible interphase layer is present and the microstructures of each layer are the same as those of their neat polymers. Understandably, 
the contact time allowing for interdiffusion is extremely short in the 2L, and thus hardly affects the morphology and microstructures (crystal, orientation, etc.) of constituent layers, as confirmed by the microstructural studies above. However, spectra for multilayered systems deviate positively from the predictions with increasing number of layers. Interestingly, the value of $\varepsilon^{\prime}$ is continuously increased with number of layers increasing up to 32, and slightly reduced with further increasing layers. Wherein, 32L shows the maximum storage permittivity. It is understood that interfacial diffusion becomes more significant with the prolonged contact time by increasing the number of layers. Logically, the strong dependence of permittivity on the number of layers should be thus attributed to microstructural changes stemming from the interdiffusion among layers, as well as the layer multiplication during the coextrusion process that involves compression and expansion flows. As demonstrated above, the $\alpha$-phase crystal of PVDF is the main crystalline form in all multilayer films under investigation. The increase in $\varepsilon^{\prime}$ with the number of layers is primarily ascribed to the reduced crystallinity. As aforementioned, inter-mixing between PMMA and PVDF at their interfaces increasingly suppresses the crystallization of PVDF, thereby leading to more dipoles in amorphous regions, ${ }^{47}$ as confirmed by the increased peak intensity of $\alpha_{\mathrm{a}}$ relaxation (Figure $5 \mathrm{~b}-\mathrm{c}$ ). Meanwhile, various imperfections, including celiae, chain loops at the lamellar surface, chain rotations and twisting within crystals, and discontinuities, are raised by decreasing crystallization, which also increases the dipoles. ${ }^{43}$ As a result, the sum of dipole polarizations is enhanced with the decreased crystallization, thereby improving the storage permittivity. Apart from the increased dipoles with the reduced crystallization, the slightly enhanced edge-on orientation of PVDF lamellae by reducing the layer thickness via layer multiplication should also contribute to the increase in permittivity. This is because of the fact that upon orientation the alignment of the $c$-axis of crystals along the film in-plane direction benefits the preferential orientation of dipole moments parallel to the external electric field, which can increase the dielectric constant. ${ }^{2,} 48$ However, when the number of layers is further increased to 2048, crystal lamellae become less oriented and more imperfect due to the substantial interdiffusion across the layers and the geometrical confinement (Figure 3d,h): this structural result could explain the slightly lower storage permittivity for nanolayered films than their microlayered counterparts (Figure 5a). Furthermore, this variation could be further supported by the closer dielectric responses of a 2048L to its equivalent blend (Figure 5a), where the latter system is considered to be more interdiffused. Nevertheless, the storage permittivity for the 2048L film is still slightly larger than that of blend, which is due to the presence of multilayered morphology and edge-on oriented crystals in the former. In addition to those influences of crystal microstructures and orientations within layers, the presence of the diffuse interphases generated from interdiffusion between component layers could also influence the observed dielectric responses. This concern will be resolved in the following section. 


\subsection{Diffuse interphases in multilayers revealed by dielectric relaxation spectroscopy}

It is surprising to note that interfacial diffusion at the interface during coextrusion remarkably altered the microstructure of constituent layers, which led to different dielectric properties of multilayer films. To further exclusively probe the contribution from diffuse interphases, we further studied the dielectric relaxation behaviors of PVDF/PMMA multilayer films in the melt state. Figure 6 presents the frequency dependence of dielectric spectra recorded at $220^{\circ} \mathrm{C}$ for multilayer films. For comparison, corresponding spectra for neat polymers and the PVDF/PMMA (50/50) blend were also included therein. Besides, the theoretical spectra of multilayer films without any interphase layers are also plotted in Figure 6a-c (dashed lines) based on Eq. (2). To quantitatively analyze the dielectric responses, the experimental permittivity spectra were fitted with a nonlinear least squares formula according to the Havriliak-Negami (HN) equation: ${ }^{46,49}$

$$
\varepsilon^{*}=\varepsilon^{\prime}-i \varepsilon^{\prime \prime}=\varepsilon_{\infty}+\frac{\sigma_{D C}}{i \varepsilon_{0} \omega}+\sum \frac{\Delta \varepsilon}{\left[1+\left(i \omega \tau_{H N}\right)^{\alpha}\right]^{\beta}}, \quad i=(-1)^{1 / 2}
$$

where $\varepsilon^{*}$ is the complex dielectric permittivity, $\varepsilon_{\infty}$ represents the value of $\varepsilon^{\prime}$ at infinite frequency, $\sigma_{D C}$ is the direct current (DC) conductivity, $\varepsilon_{0}$ is the vacuum permittivity, $\omega$ is the angular frequency $(\omega=2 \pi f), \Delta \varepsilon$ is the relaxation strength of a relaxation process $\left(\Delta \varepsilon=\varepsilon_{s}-\varepsilon_{\infty}\right.$ with $\varepsilon_{s}$ being the limited low-frequency permittivity), and $\alpha$ and $\beta(0<\alpha, \beta \leq 1)$ are the shape parameters describing the symmetry and asymmetry broadening of relaxation peaks, respectively; the characteristic $\mathrm{HN}$ relaxation time $\tau_{H N}$ is related to the loss peak relaxation time $\tau_{\max }$ by the following equation:

$$
\tau_{\max }=\tau_{H N}\left[\sin \left(\frac{\alpha \beta \pi}{2 \beta+2}\right)\right]^{1 / \alpha}\left[\sin \left(\frac{\beta \pi}{2 \beta+2}\right)\right]^{-1 / \alpha}
$$

Besides, the experimental plots of dielectric loss modulus $M^{\prime \prime}$ versus frequency were also fitted using the HN equation satisfied in the modulus formalism: ${ }^{46}$

$$
\begin{aligned}
M^{*} & =\frac{1}{\varepsilon^{*}}=\frac{\varepsilon^{\prime}}{\varepsilon^{\prime 2}+\varepsilon^{\prime 2}}+i \frac{\varepsilon^{\prime \prime}}{\varepsilon^{\prime 2}+\varepsilon^{\prime 2}}=M^{\prime}+i M^{\prime \prime} \\
& =M_{\infty}+\sum \frac{\Delta M}{\left.1+\left(-i\left(\omega \tau_{H N-M}\right)^{-1}\right)^{\gamma}\right]^{5}}
\end{aligned}
$$

where $\Delta M=M_{s}-M_{\infty}, M_{s}=1 / \varepsilon_{s}, M_{\infty}=1 / \varepsilon_{\infty}, \gamma$ and $\delta$ describe the width and asymmetry of the spectra, and $\tau_{H N-M}$ is the HN relaxation time which can be also related to relaxation time $\tau_{\max }$ in $M^{\prime \prime}$ spectra by Eq. (6). The fitted curves are plotted in Figure 6a-c, and fitting parameters are reported in Table 4. 
(a)

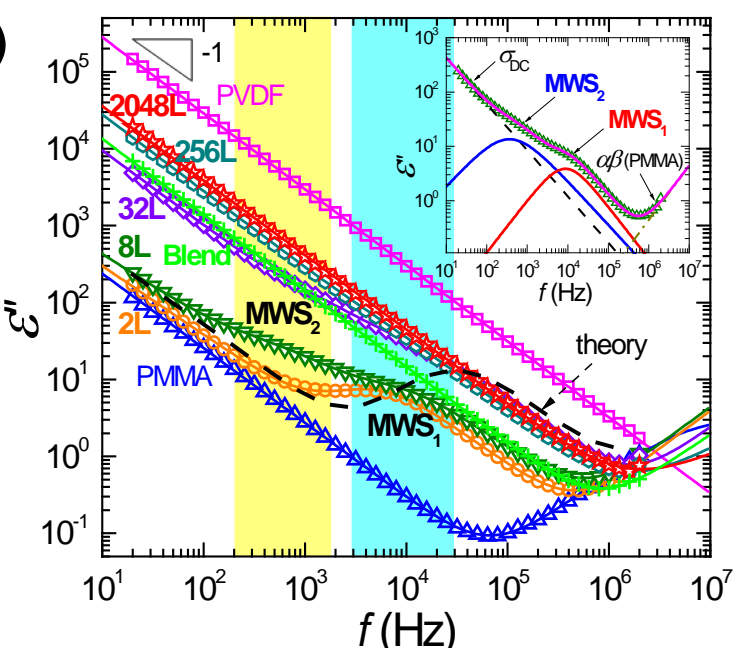

(b)

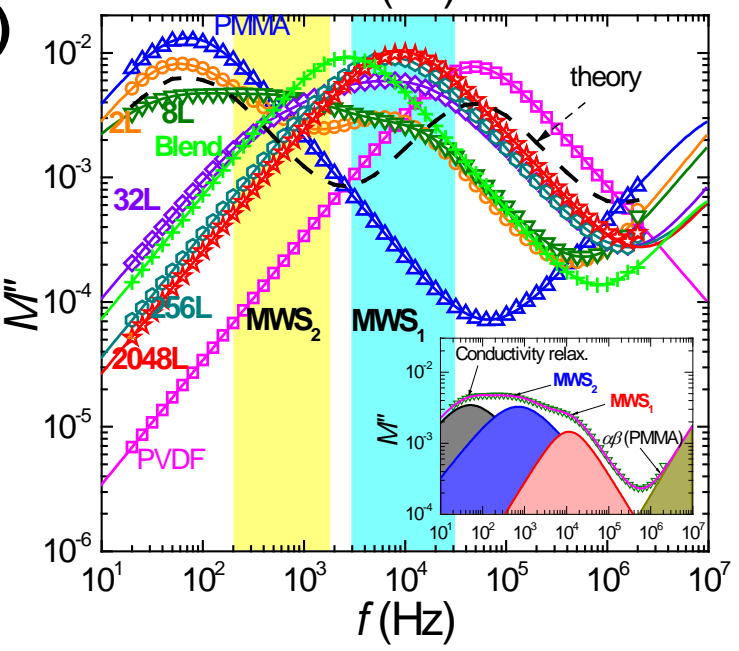

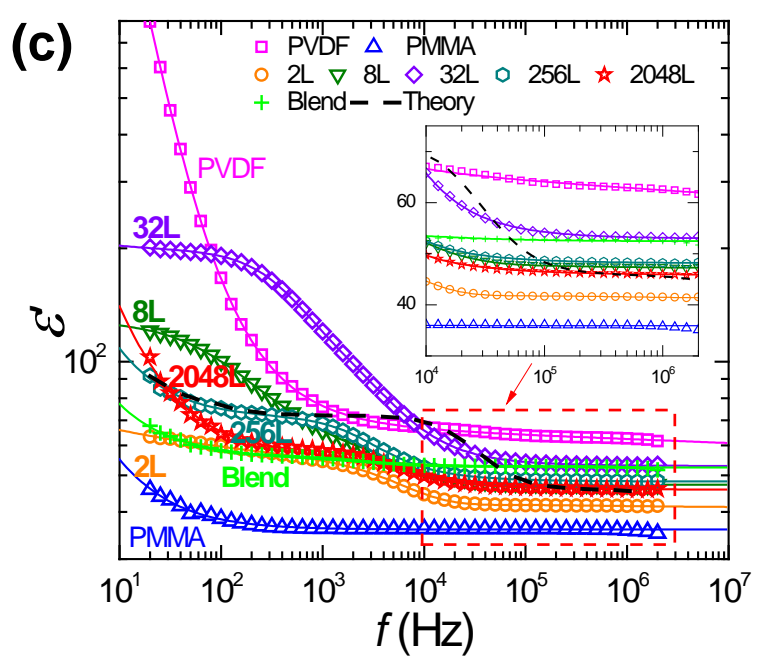

(d)

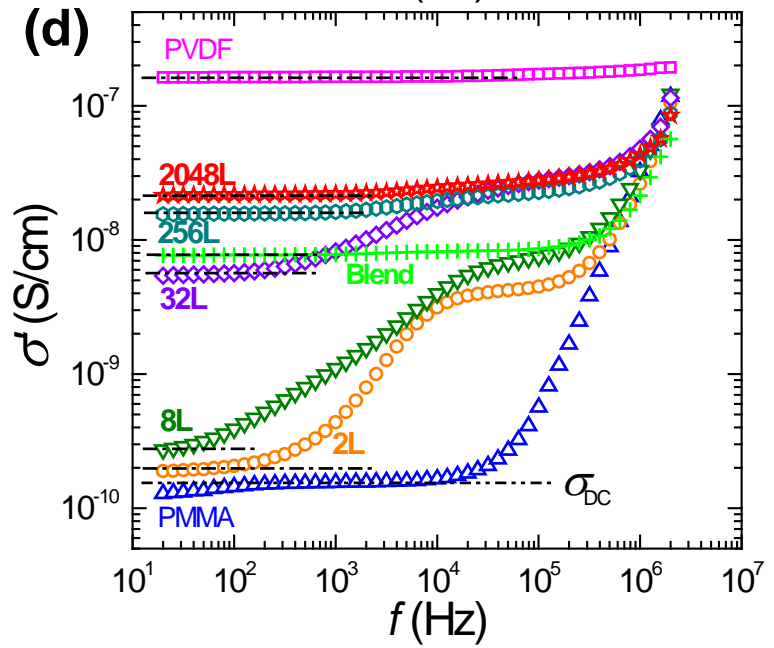

Figure 6. Dielectric spectra recorded at $220{ }^{\circ} \mathrm{C}$ for PVDF/PMMA multilayers, neat polymers (monolayers) and PVDF/PMMA blend (50/50): (a) Loss permittivity spectra, (b) loss modulus spectra, (c) storage permittivity $\varepsilon^{\prime}$ spectra, and (d) real part of complex conductivity $\sigma^{\prime}$ spectra $\left(\sigma^{\prime}=\omega \varepsilon_{0} \varepsilon^{\prime \prime}\right)$. The insets in (a) and (b) are the respective schematic deconvolutions of loss permittivity and loss modulus spectra as obtained from the fit for $8 \mathrm{~L}$, and inset in (c) shows the enlarged image of the selected region. Solid lines in $(\mathrm{a}-\mathrm{c})$ are overall fits to the Havriliak-Negami equation. Dashed lines in $(\mathrm{a}-\mathrm{c})$ are theoretical predictions for layered dielectrics without interphase layers by Equations (2-4). Dash-dotted lines in (d) indicate the DC conductivity.

It is imperative to mention that the chosen temperature $\left(220^{\circ} \mathrm{C}\right)$ for dielectric investigation here is far above the $T_{g}$ of constituent polymers (Table 1) and also higher than the equilibrium melting temperature of PVDF (i.e., $210{ }^{\circ} \mathrm{C}$ ). ${ }^{50}$ Effects of crystals and crystal-amorphous interphases on the dielectric responses are therefore suppressed herein. It is also known that PVDF and PMMA are respectively identified as dielectric type-B and type-C polymers, ${ }^{46}$ thus the normal mode relaxation related to the end-to-end vector of whole chains is also dielectrically invisible in the DRS spectra. As for neat polymers, a linearly abrupt increase in $\varepsilon^{\prime \prime}$ with decreasing frequency characterized by a relation $\varepsilon^{\prime \prime} \sim \omega^{-1}$ can be observed (Figure 6a), which is mainly contributed by DC conductivity (see more dielectric spectra of PVDF and PMMA in 
Figure S5). ${ }^{51,52}$ As for PVDF, DC conductivity almost dominates the $\varepsilon^{\prime \prime}$ spectrum at $220{ }^{\circ} \mathrm{C}$ and no segmental or molecular relaxation peaks are captured in the measured frequency range. As for PMMA, the dielectric relaxation has been observed as the contribution from both DC conductivity at lower frequencies, and the merged $\alpha \beta$ relaxation at frequencies above $10^{5} \mathrm{~Hz}^{43}$ In another representation of dielectric relaxations, the spectra of loss modulus $\left(M^{\prime \prime}\right)$ for PVDF and PMMA are displayed in Figure 6b. As can be clearly seen for PMMA, aside from the $\alpha \beta$-relaxation peak, another peak appears at low frequencies $\left(10^{2}\right.$ $\mathrm{Hz}$ ), which corresponds to the conductivity relaxation with characteristic relaxation time $\tau_{\max }$ defined by $2 \pi f_{\max } \tau_{\max }=1$. The conductivity relaxation peak describes the mobility of charge carriers (catalyst, impurity ions, surfactants, etc.) transporting through the entire physical dimensions of the sample. By contrast, the conductivity relaxation peak of PVDF emerges at higher frequencies $\left(10^{4}-10^{5} \mathrm{~Hz}\right)$ (Figure 6b), which is distinguishable from that of PMMA, suggesting the higher mobility of charge carriers in PVDF (see the conductivity relaxation time in Table 4). Meanwhile, the conductivity relaxation peak observed in $M^{\prime \prime}$ spectra for PVDF/PMMA (50/50) blend is located at frequencies between those of two components.

Table 4. HN parameters obtained by the fitting of the dielectric spectra at $220^{\circ} \mathrm{C}$

\begin{tabular}{|c|c|c|c|c|c|c|c|c|c|c|c|}
\hline & \multicolumn{2}{|c|}{ Conductivity relax. } & \multicolumn{4}{|c|}{$\mathrm{MWS}_{1}$} & \multicolumn{4}{|c|}{$\mathrm{MWS}_{2}$} & \multirow[b]{2}{*}{$\varepsilon_{\infty}$} \\
\hline & $\begin{array}{c}\sigma_{D C} \\
(\mathrm{~S} / \mathrm{cm})\end{array}$ & $\begin{array}{l}\tau_{\max } \\
(\mathrm{s})\end{array}$ & $\Delta \varepsilon$ & $\alpha$ & $\beta$ & $\begin{array}{l}\tau_{\max } \\
(\mathrm{s})\end{array}$ & $\Delta \varepsilon$ & $\alpha$ & $\beta$ & $\begin{array}{l}\tau_{\max } \\
(\mathrm{s})\end{array}$ & \\
\hline $2 \mathrm{~L}$ & $1.10 \times 10^{-10}$ & $2.5 \times 10^{-3}$ & 13.07 & 0.96 & 1 & $2.41 \times 10^{-5}$ & - & - & - & - & 41.23 \\
\hline $8 \mathrm{~L}$ & $1.28 \times 10^{-10}$ & $3.10 \times 10^{-3}$ & 8.20 & 0.96 & 1 & $1.40 \times 10^{-5}$ & 36.35 & 0.81 & 1 & $2.24 \times 10^{-4}$ & 47.18 \\
\hline $32 \mathrm{~L}$ & $4.72 \times 10^{-9}$ & $3.13 \times 10^{-3}$ & 22.76 & 0.89 & 1 & $1.18 \times 10^{-5}$ & 89.82 & 0.87 & 1 & $5.76 \times 10^{-5}$ & 52.97 \\
\hline $256 \mathrm{~L}$ & $1.52 \times 10^{-8}$ & $5.28 \times 10^{-5}$ & 9.33 & 0.89 & 0.86 & $1.0 \times 10^{-5}$ & 11.30 & 0.96 & 1 & $3.88 \times 10^{-5}$ & 48.17 \\
\hline 2048L & $1.75 \times 10^{-8}$ & $1.75 \times 10^{-5}$ & - & - & - & - & - & - & - & - & 45.86 \\
\hline PVDF & $1.49 \times 10^{-7}$ & $3.36 \times 10^{-6}$ & - & - & - & - & - & - & - & - & 60.94 \\
\hline PMMA & $1.10 \times 10^{-10}$ & $2.22 \times 10^{-3}$ & - & - & - & - & - & - & - & - & 35.93 \\
\hline Blend & $7.10 \times 10^{-9}$ & $5.89 \times 10^{-5}$ & - & - & - & - & - & - & - & - & 52.45 \\
\hline
\end{tabular}

Interestingly, the dielectric responses for multilayered systems at $220{ }^{\circ} \mathrm{C}$ depicted in Figure 6 show a strong dependence on the nominal number of layers. An increase in the $\varepsilon^{\prime \prime}$ intensity at lower frequencies with increasing the number of layers is clearly noticed, which is because of the improved conductivity from the increased diffusion of PVDF segments (more conducting component) via interlayer diffusion (see $\sigma^{\prime}$ spectra in Figure 6d). Also, the easier charge transport with reducing layer thickness also contributes to the increase in conduction loss in the $\varepsilon^{\prime \prime}$ spectra. ${ }^{16}$ Surprisingly, for a two-layer film (2L), a noticeable shoulder-like peak emerges in $\varepsilon^{\prime \prime}$ at the frequency region around $10 \mathrm{kHz}$, which cannot be assigned to any segmental or molecular relaxations of components. This additional relaxation in 2L can be more clearly distinguished from the $M^{\prime \prime}$ spectrum (Figure 6b). However, this peak is not observed for the 
PVDF/PMMA (50/50) blend featuring a homogeneous structure in the melt (Figure 6a,b). As addressed above, normal mode relaxation is dielectrically inert for components, and electrode polarization is suppressed at $220^{\circ} \mathrm{C}$, as well as effects of crystals and crystal-amorphous interphases. Instead, the emerging relaxation is attributed to the relaxation of charge carriers at the interface within the material, i.e., interfacial Maxwell-Wagner-Sillars (MWS) polarization. ${ }^{46} \mathrm{~A}$ necessary condition for the MWS process is a sufficient contrast in the $\varepsilon^{\prime}$ and/or $\sigma_{D C}$ between two media; under an alternating electric field, space charges are thus blocked near the interface and locally relax therein, leading to an interfacial polarization/relaxation. ${ }^{46}$ As for the 2L PVDF/PMMA film under this study, it is understandable that the MWS process occurs considering the substantial contrast in the $\sigma_{D C}$ by three orders of magnitude and also in $\varepsilon^{\prime}$ by 30 between two components at $220{ }^{\circ} \mathrm{C}$ (Figure 6c-d and Table 4). Unlike a structurally homogeneous blend where there is no space charge blocked within the material, the layered system of $2 \mathrm{~L}$ with a prominent interface effectively traps the space charges at the interface between PVDF and PMMA layers under an external alternating electric field, manifesting in the substantial MWS relaxation. In other words, dielectric spectroscopy can effectively probe the presence of interfaces in the layered structures. The presence of interfacial MWS relaxation in layered systems could be also predicted, as shown by the theoretical spectra in Figure 6a-b, where the characteristic relaxation frequency of MWS peak could be extracted according to:

$$
f_{M W S}=\frac{\phi_{A} \sigma_{D C, A}}{2 \pi \varepsilon_{0}\left(\phi_{A} \varepsilon_{A}+\phi_{B} \varepsilon_{B}\right)}
$$

with $A$ being the more conducting layer (i.e., PVDF), and $\varepsilon_{A}$ and $\varepsilon_{B}$ their dielectric constant at the studied temperature. ${ }^{44,46}$ Note that $f_{\max }$ is highly contingent on the concentration ratio and dielectric properties of two constituent layers. The value of $f_{\max }$ for PVDF/PMMA (50/50) multilayer without interphase layers is calculated to be $c a .2 .5 \times 10^{4} \mathrm{~Hz}$. The slightly lower $f_{\mathrm{Mws}}$ for the $2 \mathrm{~L}$ than the prediction is likely due to the presence of a thin interphase from interdiffusion and also somewhat survived chain orientation (along the film in-plane direction) in the melt from coextrusion. This could suppress the localized movement of charges parallel to the electric field and thus shift the MWS relaxation peak to the lower frequencies.

Another striking fact to discuss is the interfacial relaxations varying with the number of layers for these multilayer samples (Figure 6a-b). Different from 2L and also the theoretical prediction, systems from 8 to 256 layers, especially for the 8L, clearly display bimodal MWS processes in the loss spectra ( $\varepsilon^{\prime \prime}$ and $\left.M^{\prime \prime}\right)$, where one MWS peak (we denote this as $\mathrm{MWS}_{1}$ ) appears at the frequency region identical to that of $2 \mathrm{~L}$, and the other $\left(\mathrm{MWS}_{2}\right)$ emerges at the lower frequencies $\left(10^{3} \mathrm{~Hz}\right)$ (see schematic deconvolution of the relaxations in insets of Figure 6a-b). The occurrence of bimodal MWS relaxations in those layered structures is supposed to be resulting from the differences in the extent of interdiffusion with various contact times and diffuse interphases. Herein, before further analysis of dielectric relaxations, it is 
necessary to first understand the interdiffusion and diffuse interphase development in PVDF/PMMA multilayer films from the coextrusion process. As schematically illustrated by Figure 7a, interdiffusion between two laminar melts (A/B) occurs immediately when they come into contact during the combination in the feedblock, and subsequently creates an initial interphase $\left(I_{1}\right)$ composed of both component segments. Then, each multiplier element multiplies the layers and subsequently generates a new interphase, such that after $n$ multipliers one can fabricate a $2^{n+1}$ layered product containing $\left(2^{n+1}-1\right)$ interphases. Simultaneously, the older interphases expand with time via interdiffusion until the melt is quenched after exiting the die. Understandably, the older and new interphases are different due to the difference in the amount of interdiffusion with different contact times. That is to say, both older interphases with larger thicknesses, and the new ones with smaller thicknesses coexist in the final films. Logically, compared to a new interphase, an older interphase more effectively reduces the thicknesses of its outer bulk layers due to the larger length scale of interdiffusion at a longer contact. This can explain the bimodal interfacial MWS processes observed in the microlayers (8-256L). As shown by the scheme in Figure 7a, the 8L film, taken as an example, possesses three types of interphases $\left(I_{1}, I_{2}\right.$ and $\left.I_{3}\right)$ generated at different stages during coextrusion: the earliest formed $I_{1}$ has the thickest scale, whereas the latest created $I_{3}$ has the thinnest scale, together with an intermediate $I_{2}$ formed at the time between those of $I_{1}$ and $I_{3}$. It is also worth noting that the interdiffusion between PVDF and PMMA is of fast-mode mechanism, i.e., asymmetric diffusion. ${ }^{27}$ Considering the configuration of the multilayered structure, we can thus imagine that the concentration ratio of two layers outside the older interphases $I_{1}$ and $I_{2}$, especially $I_{1}$, deviates more from the initial composition of 50/50, whereas that for last formed interphase $I_{3}$ should almost retains 50/50 due to the relatively negligible interdiffusion. Under an external electric field, a number of space charges blocked at the interfacial region around $I_{3}$, of which the properties are similar to the interphase of $2 \mathrm{~L}$, and thus relax at the same frequency region as the latter (see $\mathrm{MWS}_{1}$ in Figure 6a,b and 7b). By contrast, the interfacial regions of earlier formed $I_{1}$ and $I_{2}$ have the larger capacity to trap space charges due to the bigger contrast in the component concentrations of outer layers, as compared to that in $I_{3}$; therefore, they display the MWS processes with a higher relaxation strength $(\Delta \varepsilon)$ at the lower frequencies (Figure 6a-b). The broader relaxation peak of $\mathrm{MWS}_{2}$ than that of $\mathrm{MWS}_{1}$ for $8 \mathrm{~L}$ is due to the merging of MWS relaxations at the regions of $I_{1}$ and $I_{2}$, as evidenced by the smaller shape factor $\alpha$ of $\mathrm{MWS}_{2}$ (see Table 4 and Figure 7b). Besides, the presence of inhomogeneous layer thicknesses might also contribute to the broadening of $\mathrm{MWS}_{2}$ relaxation.

Meanwhile, it is also noteworthy in Figure 6a,b that MWS dispersions are gradually merged together with conductivity relaxation and vanishes with reduced relaxation strength $(\Delta \varepsilon)$ by further increasing the number of layers beyond 256 (Table 4); thereafter they are hardly discerned with the number of layers reaching 2048 where the layer thickness is on nanoscale. The reason underlying the suppressed MWS processes is two-fold: (1) the presence of substantial interdiffusion minimizes the contrast in the 
component concentration and properties of two neighboring layers and therefore suppresses the MWS relaxations; (2) the increasingly negative deviation of layer thickness from the length scale over which charge carriers accumulate, resulting in much more charge hopping through all the layers than being trapped at the interfaces. ${ }^{45}$ The second concern could be validated by the continuously increased DC conductivity with the number of layers in Figure 6d. Nevertheless, it is also important to note that the relaxation behavior for a nanolayered 2048L film still differs from that of a homogeneous blend, despite the undetectable MWS process. We note that the loss relaxation peak in the $M^{\prime \prime}$ spectrum of 2048L is broader than that of blend. Therein, the shape factor $\alpha$ for the $M^{\prime \prime}$ peak of 2048L is determined to be 0.97, which deviates from " 1 ” of a typically homogeneous medium and also smaller than 0.99 of the blend. The broader the $M^{\prime \prime}$ peak in 2048L is ascribed to the merging of conductivity relaxation and a potential MWS process, as well as the inhomogeneous distribution of space charges. Therefore, the broadening of $M^{\prime \prime}$ peak in 2048L clearly indicates the structural heterogeneity due to the presence of multilayered structure and layer-layer interphases, which is in good agreement with the results from structural investigations earlier.

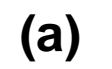

Combination

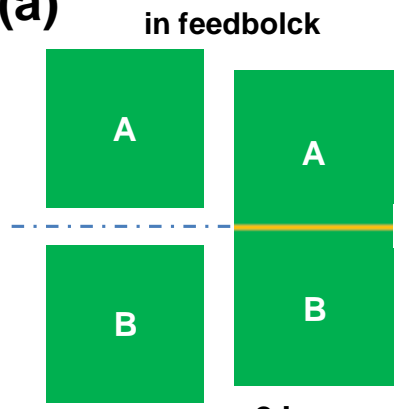

2 layers
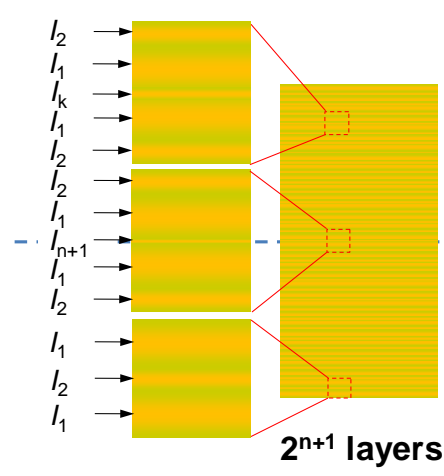

$1^{\text {st }}$ multiplier

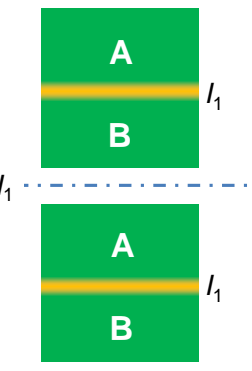

$\mathrm{n}^{\text {th }}$ multiplier

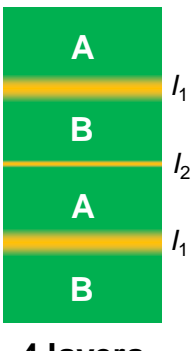

4 layers

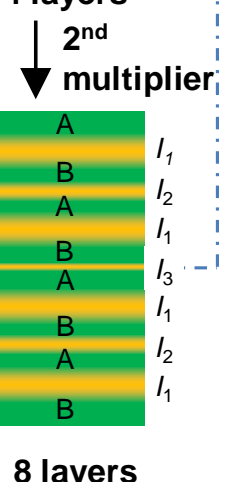

(b)

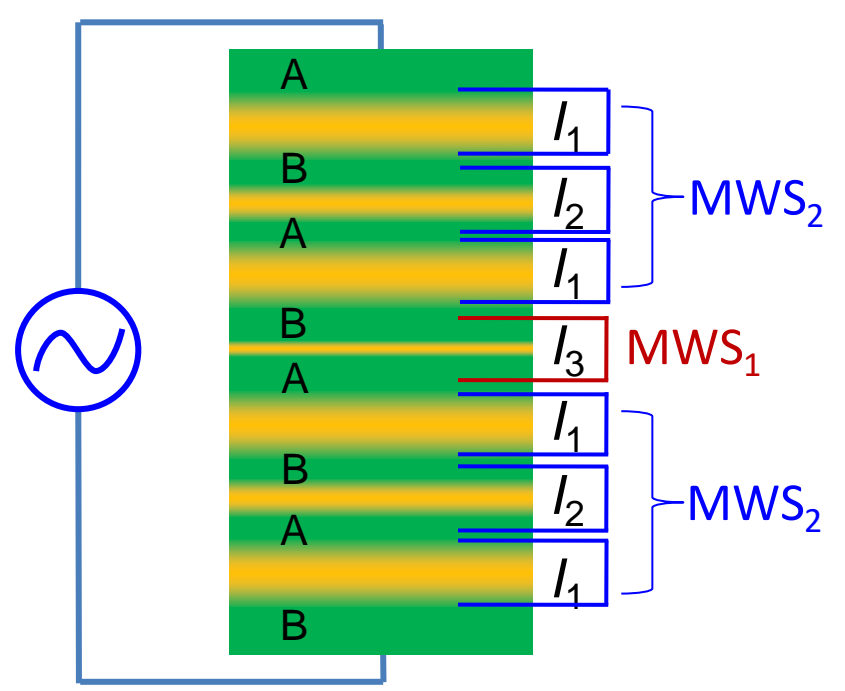

Figure 7. (a) Proposed schematic illustration showing the interdiffusion and development of diffuse interphases during the coextrusion process for a compatible multilayered A/B system. "I" stands for the interphase shown by the bright regions in the schematic layered structures. (b) Scheme explaining the bimodal MWS relaxations for multilayered PVDF/PMMA films subjected to an alternating electric field by taking an 8L film as an example (A: PVDF, B: PMMA). 
Additionally, storage permittivity measured at $220^{\circ} \mathrm{C}$ for multilayer films shows the same dependence of number of layers as that tested at the solid state. Notably, the value of the permittivity at infinite frequency $\left(\varepsilon_{\infty}\right)$ for multilayered samples is steadily increased with the number of layers $(N)$ increasing up to 32, but reduced with further increasing $N$ towards nanolayered structures (Figure 6c and Table 4). As aforementioned, storage permittivity for multilayer systems in the melt is primarily contributed by interfacial polarization, as other polarizations are greatly minimized. The change in $\varepsilon^{\prime}$ with the number of layers for multilayered samples at $220{ }^{\circ} \mathrm{C}$ should be ascribed to the presence of interfaces. The more interfaces lead to more interfacial polarization, thus contributing to the overall storage permittivity. ${ }^{2}$ This could be evidenced by the case of 32L, where the prominent interfacial polarization (MWS process) remarkably increases the magnitude of $\varepsilon^{\prime}$ especially at lower frequencies. By contrast, for the films with a larger number of layers, the amount of interfaces is minimized by the substantial interdiffusion at a longer contact time. Interfacial polarization is thus reduced, leading to a lower permittivity with the amplitude closer to that of a fully diffused blend (Figure 6c).

\subsection{Length scale (thickness) of diffuse interphases in multilayers}

To gain further insights into the length scale (thickness) for the diffuse interphases in PVDF/PMMA multilayer films, we first examined the interfacial properties using a model stacked PVDF/PMMA bilayer assembly based on a rheological methodology (see Appendix A). ${ }^{27-29}$ The average concentration of PMMA $\left(\phi_{P M M A}\right)$ in the triggered diffuse interphase can be approximately determined by comparing the value of complex modulus $\left(G^{*}\right)$ for the PVDF/PMMA sandwich with those of PVDF/PMMA blends with various compositions as described elsewhere, ${ }^{26,27}$ assuming the interphase to be a homogeneous blend with the composition changing with the diffusion time. Figure 8a illustrates the average concentrations of PMMA ( $\left.\phi_{P M M A}\right)$ within the diffuse interphase as a function of healing time at $220^{\circ} \mathrm{C}$ for a PVDF/PMMA bilayer measured by oscillatory shear with angular frequency in the terminal region (i.e., under static conditions). The PVDF concentration $\left(\phi_{P V D F}=1-\phi_{P M M A}\right)$ is also plotted therein. We can notice that the PMMA concentration $\left(\phi_{P M M A}\right)$ increases rapidly at a shorter diffusion time before reaching an approximate steady value. Another important fact that can be viewed is that $\phi_{\text {PMMA }}$ remains below $50 \%$ even after a longer diffusion time, indicating the faster-moving species (PVDF) being dominated in the interdiffusion here as predicted by the fast-mode theory. ${ }^{53}$ Accordingly, a possible physical picture for the interdiffusion process is illustrated schematically by the inset of Figure 8a, where the initial interface moves towards the PMMA phase and the formed interphase is rich in PVDF segments. Figure 8b depicts the time evolution of the apparent mutual diffusion coefficient $\left(D_{m}\right)$, and the inset shows the corresponding diffuse interphase thickness $\left(h_{I}\right)$ determined at $220{ }^{\circ} \mathrm{C}$. The details regarding the determination of $D_{m}$ and $h_{I}$ are described in Appendix A and also in our previous work. ${ }^{27}$ As indicated by 
Figure 8b, $D_{m}$ is higher at a shorter time due to the diffusion dominated by short chains, and then reduces to a constant value around $6.0 \times 10^{-13} \mathrm{~m}^{2} / \mathrm{s}$ with the increased participation of long chains in the diffusion process. Meanwhile, the diffuse interphase expands upon diffusion and its thickness reaches approximately $80 \mu \mathrm{m}$ at a contact time of $45 \mathrm{~min}$.
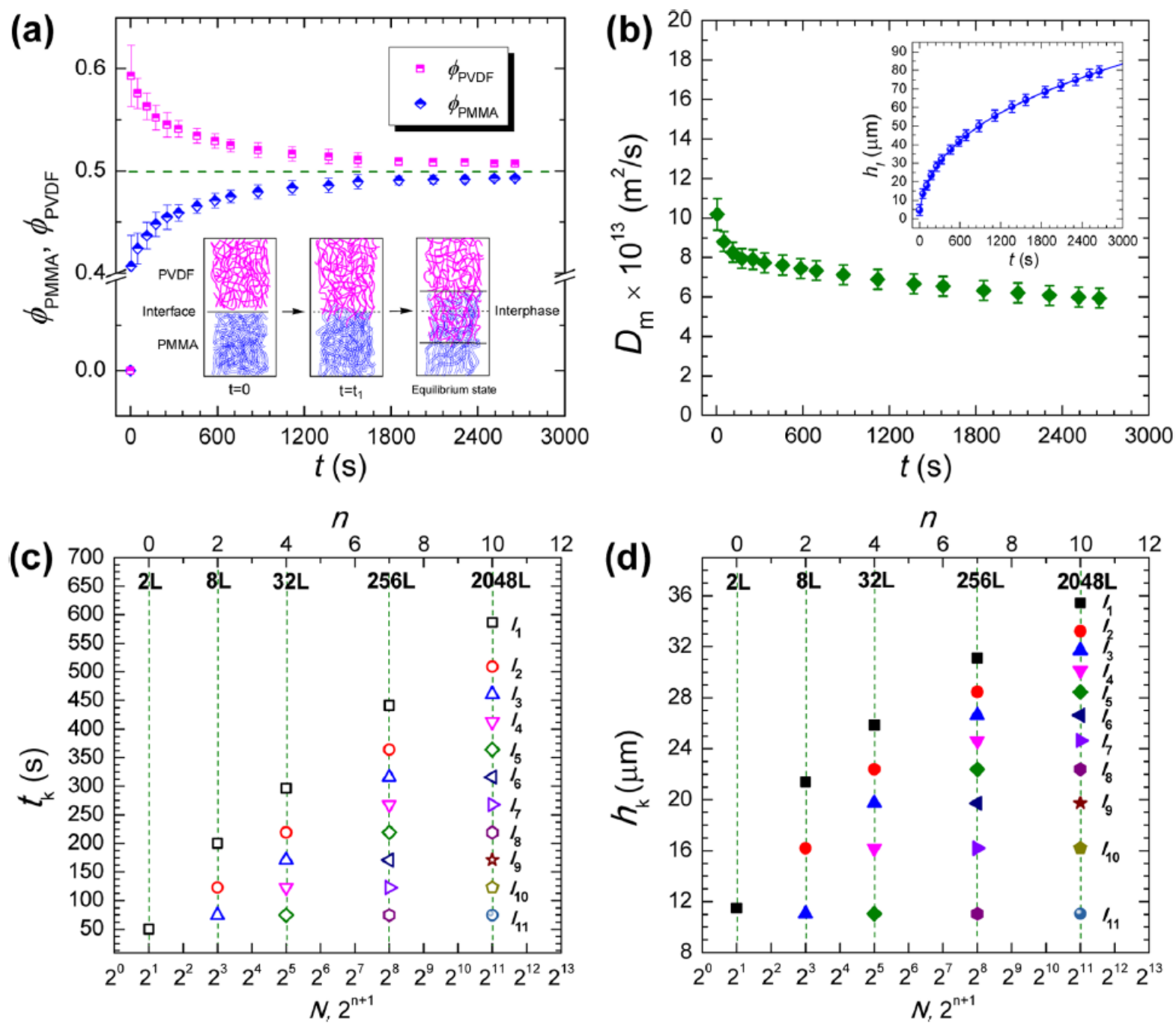

Figure 8. (a) Time evolution of the concentration profile within the diffuse interphase, and (b) time evolution of the mutual diffusion coefficient of the PVDF/PMMA sandwich assembly determined by rheological modeling at $220^{\circ} \mathrm{C}$. The dashed line in (a) indicates a concentration value of $50 \%$. The inset in (a) illustrates a proposed scheme for the interdiffusion process at the interface and the inset in (b) shows the corresponding interphase thickness vs contact time. The curve in the inset of (b) represents the least-squares fits to the data in scatters. (c, d) Calculated contact time and theoretical interphase thickness, respectively, for individual diffuse interphases in coextruded PVDF/PMMA multilayer films with various numbers of layers $(N)$ or the number of multipliers (n) $\left(N=2^{\mathrm{n}+1}\right)$.

However, under practical coextrusion flow conditions, the mutual diffusion coefficient $\left(D_{m}^{\prime}\right)$ of the PVDF/PMMA system in the melt is expected to be lower than that in static conditions because of the decelerated interdiffusion by chain orientation under flows, as well as the intermixing from interfacial 
stress. $^{28,54}$ In this sense, $D_{m}^{\prime}$ has been determined to be 0.72 times lower than $D_{m}$ of static conditions for PVDF/PMMA layered system, i.e., $D_{m}^{\prime}=0.72 D_{m}{ }^{28}$ thereby leading to the interphase thickness development under flow conditions as a function of time as follows: $h_{I}=\left(0.72 D_{m} t\right)^{1 / 2}$. During a practical coextrusion process, the extent of interdiffusion mainly depends on the contact time between neighboring layers in the melt state, assuming that the interdiffusion during the cooling process of film is negligible. As revealed earlier, multiple interdiffusion processes occur in the coextrusion because of the interfacial diffusions starting from different stages during coextrusion. A coextruder (A/B configuration) equipped with $n$ multipliers thus creates $n+1$ kinds of diffuse interphases with varying contact times and thicknesses in the final films. Figure 8c,d maps the contact time and theoretical thicknesses for individual diffuse interphases present in the coextruded PVDF/PMMA multilayers (see calculation details in Appendix B). Obviously, the contact time for a microlayer of $2 \mathrm{~L}$ is as short as $c a .50 \mathrm{~s}$, thereby leading to a single diffuse interphase of $c a .11 \mu \mathrm{m}$ in thickness that is negligible compared to the nominal thicknesses of bulk layers (Table 2). The interphase thickness can only exceed the nominal layer thickness with the number of layers increasing up to 32. It is worthwhile that in a nanolayer of 2048L, thicknesses of 2047 interphases range from 11 to $35 \mu \mathrm{m}$, which are far larger than the nominal layer thicknesses (Table 2). This agrees well with the morphological observation by TEM, where the interdiffusion prevails in the 2048L (see Figure 4e-f).

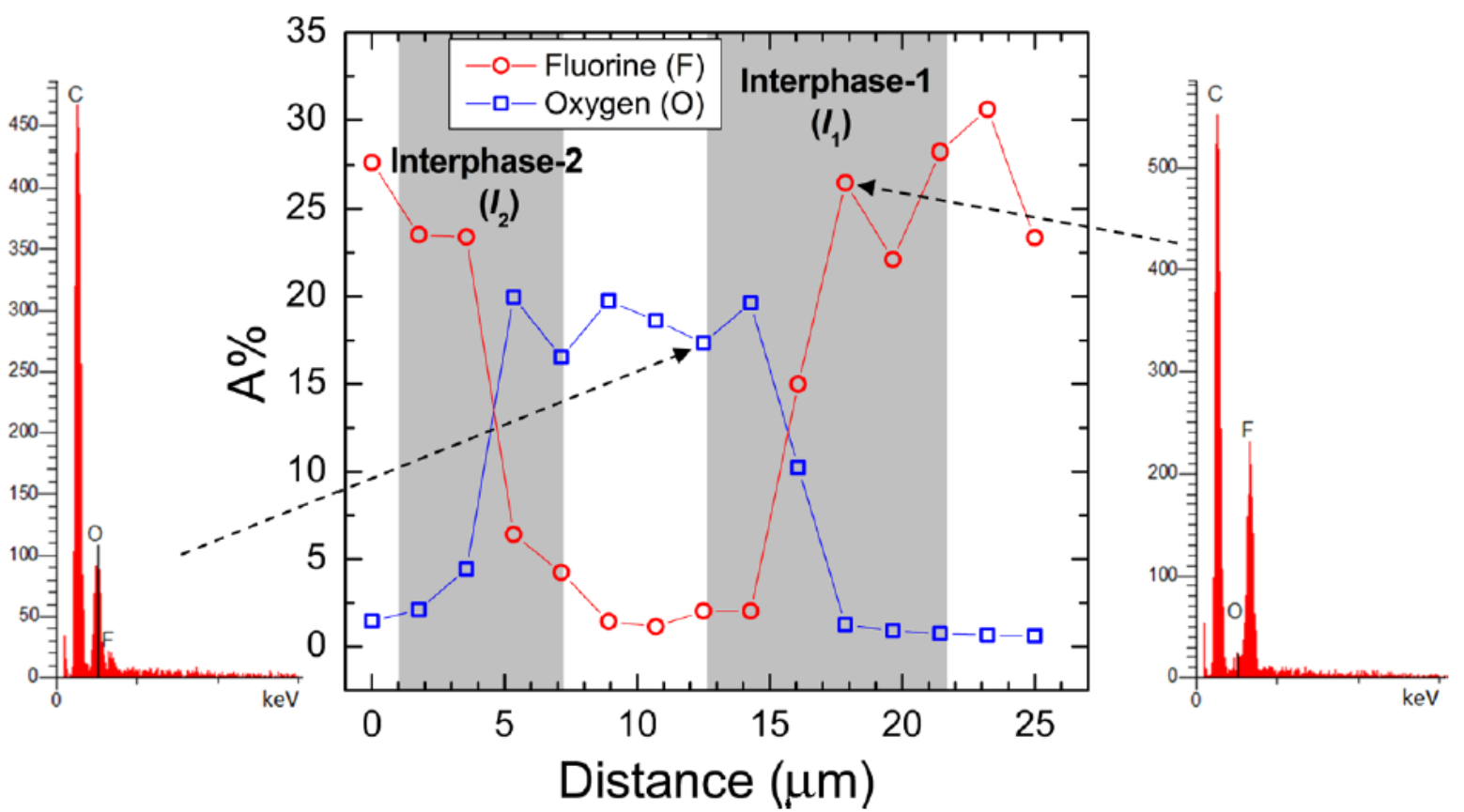

Figure 9. EDX experimentally measured concentration profile of $\mathrm{F}$ and $\mathrm{O}$ (in atomic fraction) vs measured positions for an eight-layer PVDF/PMMA film (8L). Figures appended on the left and right sides are respectively the raw data of X-ray spectrum vs energy level collected at the 8th and 11th points.

A combination of scanning electron microscopy and energy dispersive X-ray analysis (SEM-EDX) was 
further employed to experimentally determine the scale of diffuse interphases in as-coextruded multilayer films. By probing the traces of fluorine (F) element of PVDF and oxygen (O) element of PMMA compositions across the interfacial region, we could obtain a general picture of the concentration profile of two polymers versus their positions across the layer thickness. For the sake of clarity, the 8L film was representatively chosen to study the interfacial profiles. A line scan analysis including 15 points was conducted perpendicular to the layers from one PVDF-rich layer region towards a PMMA-rich layer, namely covering two interphase regions $\left(I_{1}\right.$ and $\left.I_{2}\right)$. Figure 9 displays the measured concentration profile of $\mathrm{F}$ and $\mathrm{O}$ elements along the detected route. Along the line scan path, we can clearly figure out the diffuse interphase where there is a mixture of PVDF/PMMA between two neighboring layers. As expected, two diffuse interphases with different thicknesses can be found, one is approximately $10 \mu \mathrm{m}$ thick (i.e., $I_{1}$ ), and the other is $c a$. $6 \mu \mathrm{m}$ thick $\left(I_{2}\right)$. This is in reasonable agreement with the theoretical calculation, where $I_{1}$ and $I_{2}$ correspond to ca. 21 and $16 \mu \mathrm{m}$ in thickness, respectively (Figure 8d). The deviation from the theoretical values is presumably due to the decreased thickness by flow effects during coextrusion, as well as the layer multiplication. Unfortunately, an accurate quantification of the interphase thicknesses by taking those effects into account is still challenging for this moment because many factors can influence the interdiffusion in a practical coextrusion process. ${ }^{55}$ More sophisticated instruments with a higher resolution, together with more precise mathematical models, should be employed to verify the length scale of interdiffusion, which is a matter for future work. Nevertheless, these different interphases detected by SEM-EDX here are clearly indicative of the presence of multiple interdiffusion and diffuse interphases in multilayer films. This finding remarkably corroborates the dielectric investigations above.

\subsection{Role of diffuse interphase in extensional rheological behaviors of multilayer films}

Uniaxial extensional rheology was performed on the multilayer films to probe their melt flow properties, and evaluate their processibility under flow conditions simulating the coextrusion process. Figure 10 depicts the transient extensional viscosity (stress growth coefficient) as a function of time measured at $220{ }^{\circ} \mathrm{C}$ for the coextruded neat PVDF and PMMA films. It is imperative to mention that we have measured the extensional rheology of all neat PVDF and PMMA films extruded with various numbers of multipliers as done for multilayer films and that no difference in extensional rheology was found among them. The linear viscoelastic (LVE) envelope shown by lines in Figure 10 was determined by first fitting small-amplitude oscillatory shear (SAOS) data to a multi-mode Maxwell discrete relaxation spectrum as follows:

$$
G^{\prime}(\omega)=\sum_{i=1}^{N} G_{i} \frac{\left(\omega \tau_{i}\right)^{2}}{1+\left(\omega \tau_{i}\right)^{2}}
$$




$$
G^{\prime \prime}(\omega)=\sum_{i=1}^{N} G_{i} \frac{\left(\omega \tau_{i}\right)}{1+\left(\omega \tau_{i}\right)^{2}}
$$

where $G_{\mathrm{i}}$ is the relaxation strength and $\tau_{\mathrm{i}}$ is the time constant. Then, these parameters were used to compute the LVE extensional viscosity (zero-rate limit) by following the Trouton relation for uniaxial extension viscosity, according to: ${ }^{56}$

$$
\eta_{E, 0}^{+}=\lim _{\dot{\varepsilon} \rightarrow 0} \eta_{E}^{+}(t, \dot{\varepsilon})=3 \sum_{i=1}^{N} G_{i} \tau_{i}\left(1-e^{-t / \tau_{i}}\right)
$$

For all the neat polymers, we have also demonstrated that $\eta_{E, 0}^{+}$is equal to $3 \eta_{0}^{+}$determined from a start-up shear measurement at a smaller Hencky rate of $0.01 \mathrm{~s}^{-1}$, where $\eta_{0}^{+}$is the linear viscoelastic transient shear viscosity. ${ }^{56}$ Clearly, extensional viscosities of neat polymers at small strains agree well with LVE envelope. For PMMA at all measured elongation rates, viscosities are clearly lower than the LVE domain at larger strains $\left(\varepsilon_{H}>1\right)$, showing a strain-softening behavior consistent with the classic flow behavior of linear polymers. This phenomenon is likely due to the linear architecture of PMMA chains and their extremely high stiffness with rather large monomeric friction coefficient, rendering the chain stretch difficult and limiting segment alignment. ${ }^{29}$ By contrast, as for PVDF, although the flow at all measured strain rates initially follows along the LVE envelope, significantly upward deviation from LVE occurs at shorter times when the extension rate is higher than the inverse reptation time $\tau_{\text {rep }}{ }^{-1}$, i.e., Weissenberg number $\left(W_{i}\right)$ greater than unity $\left(W_{i}>1\right)$, thus showing a strain-hardening behavior. Chain stretching in extension is supposed to be responsible for the observed strain hardening. ${ }^{57}$ The strain-hardening behavior of PVDF was also observed in our recent work. ${ }^{29}$ Strain hardening for such a linear PVDF is quite unusual, and this behavior can be presumably due to the wide molecular weight distribution (Table 1). ${ }^{56}$ Also, a small amount of high-molecular-weight fraction containing chain branching might exist resulting from the synthesis of PVDF resins, which could also give a strain hardening typical of branched polymers. ${ }^{58} \mathrm{~A}$ clear mechanism for the strain hardening of PVDF is still unclear for this moment and also not the aim of this study. 


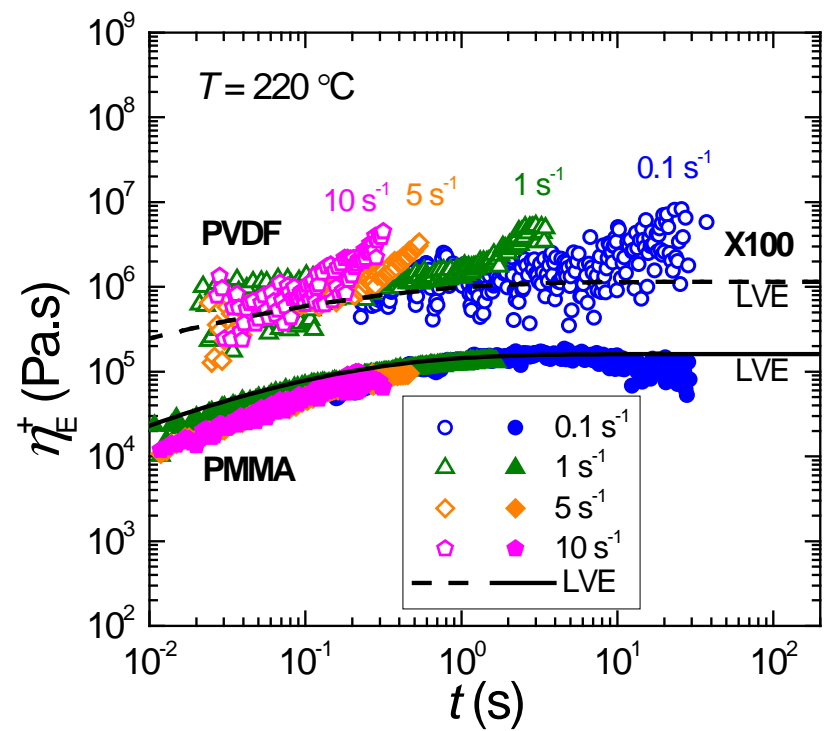

Figure 10. Extensional viscosity vs extension time at $220^{\circ} \mathrm{C}$ with extension rates varying from 0.1 to $10 \mathrm{~s}^{-1}$ for neat PVDF and PMMA films. The lines are LVE predictions. Data for PVDF are shifted vertically for clarity.

Figure 11 plots the extensional viscosities as a function of time at various strain rates for PVDF/PMMA multilayer films and the equivalent blend. Upon the uniaxial extension, the deformation rate for each layer in the multilayer structure is the same. ${ }^{29}$ The total force is the sum of the contributions of constituent layers and interphase/interlayer, which could be estimated with the following equation: ${ }^{29}$

$$
F(t)=\sigma_{A} A_{A}(t)+\sigma_{B} A_{B}(t)+\sigma_{\text {int }} A_{\text {int }}(t)
$$

where $\sigma_{i}(t)$ is the Cauchy stress of layer $i$ ( $i$ denotes component layer A, B and the interphase/interlayer). $A_{\mathrm{i}}(t)$ is the area of cross-section perpendicular to film coextrusion direction with $A_{\mathrm{i}}(t)=W_{\mathrm{i}}(t) \times H_{\mathrm{i}}(t)$, where $W_{\mathrm{i}}(t)$ and $H_{\mathrm{i}}(t)$ are the time-dependent width and thickness of a layer $i$, respectively. Therefore, the transient extensional viscosity of the total multilayer structure including interphases can be described as:

$$
\begin{aligned}
\eta_{E}^{+}(t)=\frac{F(t)}{\dot{\varepsilon} A(t)} & =\phi_{A} \frac{\sigma_{A}(t)}{\dot{\varepsilon}}+\phi_{B} \frac{\sigma_{B}(t)}{\dot{\varepsilon}}+\phi_{\text {int }} \frac{\sigma_{\text {int }}(t)}{\dot{\varepsilon}} \\
& =\phi_{A} \eta_{E, A}^{+}(t)+\phi_{B} \eta_{E, B}^{+}(t)+\phi_{\text {int }} \eta_{E, \text { int }}^{+}(t)
\end{aligned}
$$

in which $\phi_{i}$ is the volume fraction of layer $i$ with $\phi_{i}=A_{\mathrm{i}}(t) / A_{\text {total }}(t)=H_{\mathrm{i}}(t) / H_{\text {total }}(t)$. Specifically, in the absence of an interphase layer, Eq. (13) can be simplified to a simple additivity rule: ${ }^{29}$

$$
\eta_{E}^{+}(t)=\phi_{A} \eta_{E, A}^{+}(t)+\phi_{B} \eta_{E, B}^{+}(t)
$$

For comparison, $\eta_{E}^{+}$for the PVDF/PMMA multilayer without any interphase layers is calculated based on the additivity rule in Eq. (14) and shown by grey scatters in Figure 11a. The theoretical LVE envelope for PVDF/PMMA (50/50) systems is extracted from SAOS data of the equivalent blend and is also included in Figure 11a-f. As displayed in Figure 11a, the measured extensional viscosities for the 2L 
film almost follow the predictions. This observation, on one hand, validates the remarkable feasibility of the additivity rule in predicting $\eta_{E}^{+}$of the multilayer structure without interphase layers. On the other hand, it is an indication of a negligible thinner interphase present in $2 \mathrm{~L}$ due to the shorter contact time, as discussed earlier. Besides, although measured $\eta_{E}^{+}$values of the 2L substantially deviate upward from the LVE prediction, no continuous viscosity rise with time is indicated at any strain rates, i.e., the absence of viscosity maximum at the maximum achievable Hencky strain. The melt extensional behavior of 2L is therefore strain-softening. The similar extensional behavior can be noticed for 8L (Figure 11b), but the values of $\eta_{E}^{+}$no longer follow the prediction plotted in Figure 11a. Instead, the measured $\eta_{E}^{+}$values for 8L are below the predictions and closer to the LVE prediction, which indicates the presence of diffuse interphases among layers. However, when the number of layers is increased to 32, we can find a continuous viscosity rise with time at the measured strain rates, showing a strain-hardening behavior (Figure 11c). Compared with 2L and 8L films, values of $\eta_{E}^{+}$for the 32L come much closer to the LVE prediction of the blend. Same trends can be observed as the number of layers is further increased up to 2048 (Figure 11e). Another interesting fact to note is the continuous increase in the degree of strain hardening with the increase in the number of layers, despite the less upward deviation in $\eta_{E}^{+}$from the LVE prediction at the smaller strains. Specifically, the 2048L film is the most strain-hardening among the tested multilayer films (Figure 11e). 


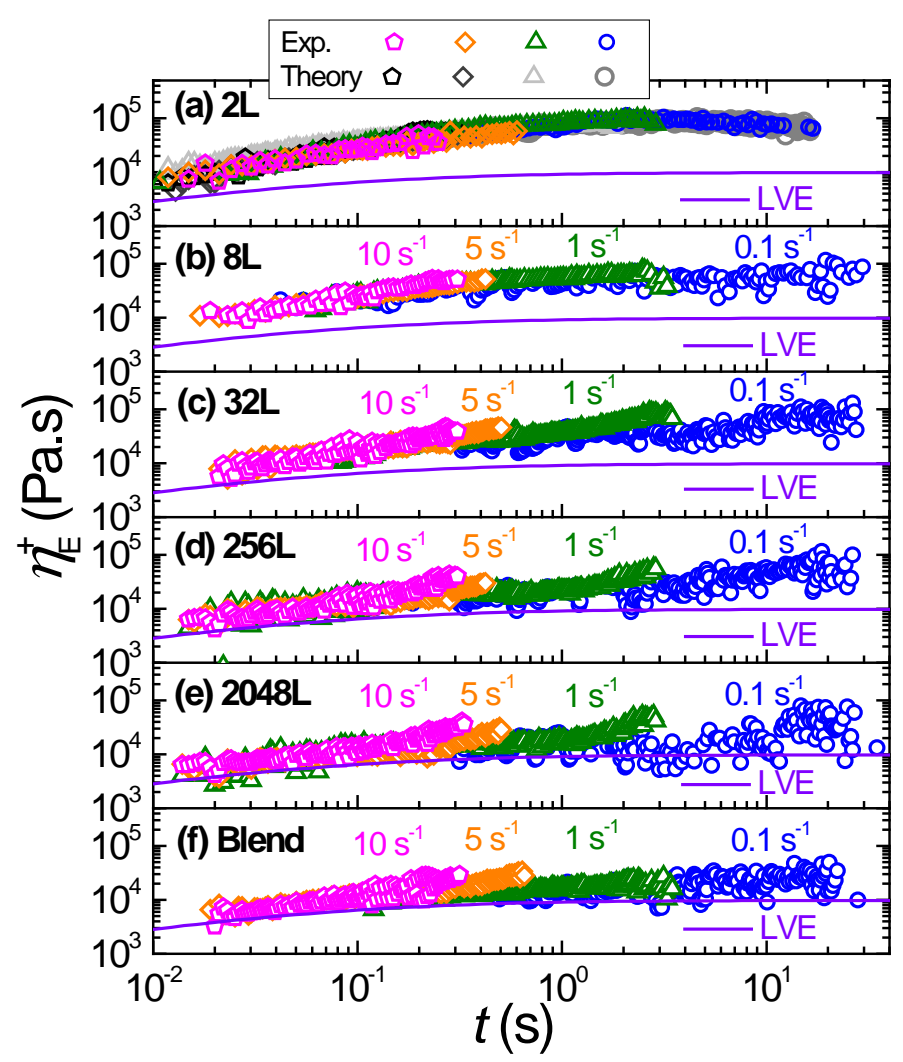

Figure 11. Extensional viscosity vs extension time at $220{ }^{\circ} \mathrm{C}$ with extension rates varying from 0.1 to $10 \mathrm{~s}-1$ for PVDF/PMMA (50/50) multilayer films and PVDF/PMMA (50/50) blend: (a) 2L, (b) 8L, (c) 32L, (d) 256L, (e) 2048L, and (f) blend. The grey scatters in the back of experimental data in (a) show the theoretical value of transient extensional viscosity for multilayer films without interphase layers. Solid lines are LVE predictions with the equilibrium equivalent PVDF/PMMA (50/50) blend.

It is surprising that increasing layers (i.e., from microlayer to nanolayer films) leads to an increasingly negative deviation in extensional viscosity at smaller strains, accompanied by an enhanced strain hardening, as compared to that in the multilayers without any diffuse interphases. These changes observed in extensional rheological properties of coextruded multilayer films are attributed to the interdiffusion and the formed diffuse interphases among neighboring layers. As discussed earlier, multiple diffuse interphases exist in the multilayer films. During a coextrusion process, coextrusion through an increasing number of multipliers increases the number of interphases, the melt contact time for interdiffusion. Meanwhile, increasing the number of layers also reduces the individual layer thickness as a consequence, and the multilayer films can be an interphase dominated material. ${ }^{19}$ For a multilayer structure, uniaxial extension deformation is realized via simultaneously stretching the layers in a direction parallel to the interface, whereby the contribution of interface/interphase and the effect of the interfacial area are thus highlighted. Logically, the diffuse interphase should play a dominant role in the extensional rheology of multilayers; the scale and inner compositions in the interphase should dictate the rheological responses. In our recent study, ${ }^{41}$ we discovered that blending PVDF with PMMA led to a negative deviation in zero-shear viscosity $\left(\eta_{0}\right)$ of blends with intermediate compositions $\left(40 \% \leq \phi_{\mathrm{PVDF}}<80 \%\right)$ due 
to the unfavorable interchain entanglements, where $\eta_{0}$ values of blends were lower than those of neat components and the mixing rule predictions. A diffuse interphase established by the interlayer mixing of component layers is similar to a blend of intermediate composition (Figure 8a). This could explain the increasingly reduced extensional viscosities at smaller strains by increasing the number of layers and also the negative deviation in $\eta_{E}^{+}$from the additivity rule predictions (Figure 11a-e). Besides, for the miscible PVDF/PMMA pair, the longer contact time in the multilayer film with a larger number of layers gives more opportunity for interdiffusion, which resembles the blending process. The extensional rheological behavior of multilayer films therefore approaches that of their equivalent blend with the increase in the number of layers (Figure 11). Here, it is also interesting to note that the extent of strain hardening of the 2048L is higher than that of its equivalent blend, despite their closer extensional viscosities (Figure 11e-f). This is because of the presence of diffuse interphases in the 2048L film and the asymmetry in the concentration profile within those interphases. As demonstrated earlier, the interdiffusion occurring in the PVDF/PMMA system is of fast-mode mechanism (Figure $8 \mathrm{a}$ ); ${ }^{27}$ therefore, the faster-moving species (PVDF) dominates in the average compositions in the triggered interphases. When the multilayer structure is subjected to extensional flows at a large deformation, the layer thickness is further reduced, the and role of these interphases among layers is thus amplified. Logically, multilayer films with a number of diffuse interphases rich in PVDF therefore manifest as macroscopic extensional strain hardening typical of the bulk PVDF. It is thus concluded that the interlayer diffusion and diffuse interphases formed in multilayered films significantly determine the rheological behaviors, especially for nanolayered systems. Such nonlinear rheological properties strongly dependent on interdiffusion and the number of layers are particularly important for industrially coextruded polymers that are subjected to processing involving extensional flows, such as coextrusion-blowing. The finding here provides a synergistic strategy to improve the processability of multilayered polymers without sacrificing the multilayered structure, by controlling the interfacial diffusion, namely, the amount and length scale of diffuse interphses among layers.

\section{Conclusions}

In this work, we revealed the role of interfacial diffusion and diffuse interphases formed in multi micro-/nanolayered polymer films prepared by forced assembly coextrusion technology, based on a compatible PVDF/PMMA system. Effects of interlayer diffusion on the morphology/microstructure development, dielectric properties and rheological behaviors of multilayer films were elaborated systematically. First, coextruded PVDF/PMMA multilayer films showed the continuous multilayered architecture, and layer morphology prevailed in all multilayer films, even for the nanolayered films where interdiffusion was significant. Interdiffusion in the presence of macromolecular confinement from rigid 
PMMA segments, coupled with the spatial/geometrical confinement from layer multiplication, was found to significantly affect the crystalline microstructure and morphology of multilayer films. These microstructural changes led to quantitatively different dielectric properties with a strong dependence on the number of layers. Second, multiple diffuse interphases with various length scales (interphase thicknesses) were revealed to exist in the multilayer films. The presence of these multiple interphases was demonstrated to be resulting from the various interdiffusions starting from different stages of the coextrusion process, and their length scales dependent on the number of layers were further mapped quantitatively. In addition, the diffuse interphases significantly altered the extensional rheological properties of the multilayer films. Intriguingly, multilayer films showed a negative deviation in extensional viscosity with the increase in the number of layers, accompanied by an enhanced strain hardening. The extent of strain hardening of the nanolayered films was even higher than that of the equivalent blend. Interdiffusion of the fast-mode mechanism and the triggered diffuse interphases with unfavorable interchain entanglements were responsible for those changes in rheological behaviors. Hopefully, the results obtained will provide a new understanding of interfaces/interphases in multilayer polymer films, toward manufacturing micro-/nanolayered polymer films with tailor-made structure and properties for some advanced applications (i.e., capacitors, energy storage devices, etc.).

\section{Appendix A}

\section{Diffuse interphase profile quantified by rheological fingerprints}

The mutual diffusion coefficient $\left(D_{m}\right)$ of the PVDF/PMMA system can be experimentally quantified by its relation with the rheological behavior of the interphase, measured by oscillatory shear with angular frequency in the terminal region (i.e., under static conditions), using a planar polymer A/B sandwich assembly with healing time (Figure 12$):^{27}$

$$
\frac{1}{G_{I}^{*}(t)}=\frac{H}{2\left(D_{m} t\right)^{1 / 2}}\left(\frac{1}{G_{s, t}^{*}}-\frac{1}{G_{s, 0}^{*}}\right)+\left(\frac{\phi_{A}}{G_{A, 0}^{*}}+\frac{\phi_{B}}{G_{B, 0}^{*}}\right)
$$

where $G_{I}^{*}(t)$ is the complex modulus of the interphase at the healing time $t$; $H$ is the total thickness of the sandwich assembly; $G_{s, t}^{*}$ and $G_{s, 0}^{*}$ are the overall complex moduli of sandwich assembly at healing times of $t$ and 0 , respectively; $G_{A, 0}^{*}$ and $G_{B, 0}^{*}$ are the respective complex moduli of polymers A and B at healing time $t=0 . D_{m}$ can be related to the monomeric friction coefficient $\left(\zeta_{b}\right)$ for the mixture as follows:

$$
\zeta_{b}=\frac{\pi^{2} k_{B} e_{b}^{2} T}{N_{b}{ }^{3} b^{4}} \frac{1}{\omega}\left[\left(\frac{8 G_{N, b}^{0}}{\pi^{2} G_{I}^{*}(t)}\right)^{2}-1\right]^{-1 / 2}
$$


where $k_{\mathrm{B}}$ is the Boltzman constant, $e_{\mathrm{b}}$ represents the stem length on the order of the gyration radius of entanglements, $N_{\mathrm{b}}$ is the number of repeat units of the blend, $b$ is the apparent effective bond length of the mixture, $\omega$ is the angular frequency, and $G_{N, b}^{0}$ represents the average plateau modulus of the interphase/blend.

The mutual diffusion coefficient can be resolved as:

$$
D_{m}=\left[\frac{(2 / 3)^{1 / 3} p}{\left(9 q+\sqrt{3} \times \sqrt{-4 p^{3}+27 q^{2}}\right)^{1 / 3}}+\frac{\left(9 q+\sqrt{3} \times \sqrt{-4 p^{3}+27 q^{2}}\right)^{1 / 3}}{2^{1 / 3} \times 3^{2 / 3}}\right]^{2}
$$

with

$$
\begin{aligned}
& p=\frac{8 \delta \omega G_{N, b}^{0}}{\pi^{2}}\left(\frac{\phi_{A}}{G_{A, 0}^{*}}+\frac{\phi_{B}}{G_{B, 0}^{*}}\right) \\
& q=\frac{8 \delta \omega G_{N, b}^{0}}{\pi^{2}} \frac{H}{2 t^{1 / 2}}\left(\frac{1}{G_{s, t}^{*}}-\frac{1}{G_{s, 0}^{*}}\right) \\
& \delta=\frac{N_{b}^{e} N_{b}^{3} b_{b}{ }^{4}}{\pi^{2} e_{b}{ }^{2}}\left(\frac{\phi_{B}}{N_{A}}+\frac{\phi_{A}}{N_{B}}\right)\left(\frac{\phi_{B}}{N_{A}}+\frac{\phi_{A}}{N_{B}}-2 \chi \phi_{A} \phi_{B}\right)
\end{aligned}
$$

in which $\chi$ is the Flory-Huggins interaction parameter and $N_{A}$ and $N_{B}$ are the number of segments per chain for polymers $\mathrm{A}$ and $\mathrm{B}$, respectively.

The resultant interphase thickness can be also estimated according to:

$$
h_{I}=2\left(D_{m} t\right)^{1 / 2}
$$

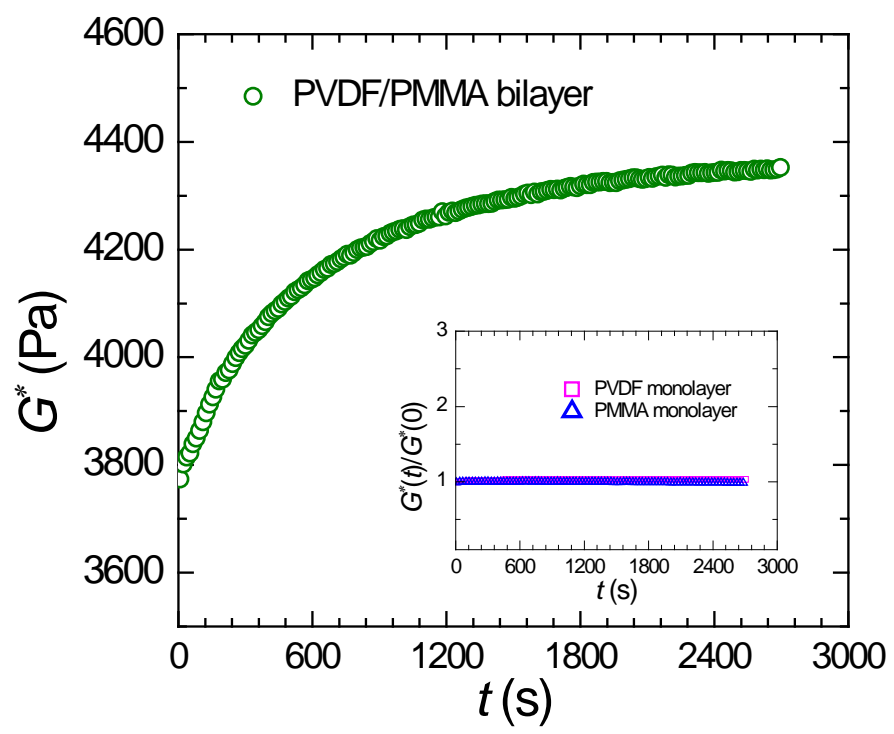

Figure 12. Complex modulus vs contact time for a PVDF/PMMA sandwich-like sample at a given frequency of $1.0 \mathrm{rad} / \mathrm{s}$, strain of 0.01 and temperature of $220^{\circ} \mathrm{C}$. The inset shows the normalized complex modulus against time for monolayers. 


\section{Appendix B}

\section{Estimation of contact time for diffuse interphases in multilayer coextrusion}

As revealed in Section 3.3, multiple diffuse interphases coexist in the coextruded films, and the contact time between adjacent layers is the total period starting from the moment of interphase creation, and then its passing through the confluent region in the multimanifold system and layer multiplying elements/multipliers (LME) in sequence until it leaves the die exit. As shown by the scheme in Figure 7a, the initial interphase $\left(I_{1}\right)$ is created in the feedblock and goes through all the confluent regions, $n$ multipliers employed and die. The contact time for interphase $I_{1}$ could be approximately estimated by relating the volumetric flow rate with the total fluid channel volume as follows:

$$
t_{1}=\frac{V_{\text {conf }}+n V_{L M E}+V_{\text {die }}}{Q_{\text {mass }}} \rho
$$

where $V_{\text {conf }}, V_{L M E}$, and $V_{\text {die }}$ are the volumes of the confluent region in the manifold of feedblock, one multiplier and die, respectively and $n$ is the number of multipliers employed. $Q_{\text {mass }}$ is the mass flow rate and $\rho$ is the apparent density of the confluent melts at the extrusion temperature.

However, a later formed interphase $I_{\mathrm{k}}(2 \leq k \leq n+1, n \geq 1)$ only goes through $(n-k+1)$ multipliers employed and die. The contact time for interphases $I_{\mathrm{k}}$ could be therefore deduced as follows:

$$
t_{k}=\frac{(n-k+1) V_{L M E}+V_{\text {die }}}{Q_{\text {mass }}} \rho
$$

\section{ASSOCIATED CONTENT}

\section{Supporting Information}

Linear viscoelasticity of the investigated polymers; thermal stability of the investigated polymers; WAXS/SAXS patterns for coextruded control films; DSC thermographs for multilayer films; and dielectric relaxation spectra of neat polymers.

\section{AUTHOR INFORMATION}

\section{Corresponding Authors}

*E-mail: khalid.lamnawar@insa-lyon.fr (K. L.).

\section{ORCID}

Bo Lu: 0000-0002-7422-853X

Khalid Lamnawar: 0000-0003-4907-0977

Guillaume Sudre: 0000-0003-3545-7046

\section{Notes}

The authors declare no competing financial interest. 


\section{Acknowledgments}

The authors acknowledge Anatoli Serghei for the fruitful discussions regarding the dielectric measurements. They would also like to acknowledge Pierre Alcouffe and Sébastien Pruvost for their kind assistance with microscope observations. They also acknowledge Baobao Chang (IPF Dresden, Germany) and Isabelle Morfin (LIPhy, Grenoble, France) for their help with X-ray scattering measurements. The WOS (WAXS detector) was funded by the French National Research Agency (ANR) under the “Investissement d'Avenir” program (Grant no. ANR-11-EQPX-0010). ARKEMA is acknowledged for providing the polymers. B.L. also acknowledges the China Scholarship Council for providing the doctoral scholarship. This research project has been financially supported by the ANR (Grant no. ANR-11-RMNP-0002) and the Auvergne-Rhône-Alpes Region Council.

\section{References}

(1) Ponting, M.; Hiltner, A.; Baer, E. Polymer Nanostructures by Forced Assembly: Process, Structure, and Properties, Macromol. Symp. 2010, 294, 19-32.

(2) Baer, E.; Zhu, L. 50th Anniversary Perspective: Dielectric Phenomena in Polymers and Multilayered Dielectric Films, Macromolecules 2017, 50, 2239-2256.

(3) Burt, T. M.; Jordan, A. M.; Korley, L. T. J. Toward Anisotropic Materials via Forced Assembly Coextrusion, ACS Appl. Mater. Interfaces 2012, 4, 5155-5161.

(4) Messin, T.; Follain, N.; Guinault, A.; Sollogoub, C.; Gaucher, V.; Delpouve, N.; Marais, S. Structure and Barrier Properties of Multinanolayered Biodegradable PLA/PBSA Films: Confinement Effect via Forced Assembly Coextrusion, ACS Appl. Mater. Interfaces 2017, 9, 29101-29112.

(5) Zheng, Y.; Dong, R.; Shen, J.; Guo, S. Tunable Shape Memory Performances via Multilayer Assembly of Thermoplastic Polyurethane and Polycaprolactone, ACS Appl. Mater. Interfaces 2016, 8, 1371-1380.

(6) Richardson, J. J.; Björnmalm, M.; Caruso, F. Technology-driven Layer-by-layer Assembly of Nanofilms, Science 2015, 348, aaa2491.

(7) Burt, T. M.; Keum, J.; Hiltner, A.; Baer, E.; Korley, L. T. J. Confinement of Elastomeric Block Copolymers via Forced Assembly Coextrusion, ACS Appl. Mater. Interfaces 2011, 3, 4804-4811.

(8) Lai, C.-Y.; Hiltner, A.; Baer, E.; Korley, L. T. J. Deformation of Confined Poly(ethylene oxide) in Multilayer Films, ACS Appl. Mater. Interfaces 2012, 4, 2218-2227.

(9) Carr, J. M.; Langhe, D. S.; Ponting, M. T.; Hiltner, A.; Baer, E. Confined Crystallization in Polymer Nanolayered Films: A review, J. Mater. Res. 2012, 27, 1326-1350.

(10) Kazmierczak, T.; Song, H.; Hiltner, A.; Baer, E. Polymeric One-Dimensional Photonic Crystals by Continuous Coextrusion, Macromol. Rapid Commun. 2007, 28, 2210-2216.

(11) Bironeau, A.; Salez, T.; Miquelard-Garnier, G.; Sollogoub, C. Existence of a Critical Layer Thickness in PS/PMMA Nanolayered Films, Macromolecules 2017, 50, 4064-4073.

(12) Liu, R. Y. F.; Jin, Y.; Hiltner, A.; Baer, E. Probing Nanoscale Polymer Interactions by Forced-Assembly, Macromol. Rapid Commun. 2003, 24, 943-948.

(13) Mackey, M.; Schuele, D. E.; Zhu, L.; Flandin, L.; Wolak, M. A.; Shirk, J. S.; Hiltner, A.; Baer, E. Reduction of Dielectric Hysteresis in Multilayered Films via Nanoconfinement, Macromolecules 2012, 45, 1954-1962.

(14) Chen, X.; Tseng, J.-K.; Treufeld, I.; Mackey, M.; Schuele, D. E.; Li, R.; Fukuto, M.; Baer, E.; Zhu, L. Enhanced Dielectric 
Properties due to Space Charge-induced Interfacial Polarization in Multilayer Polymer Films, J. Mater. Chem. C 2017, 5 , 10417-10426.

(15) Huang, H.; Chen, X.; Yin, K.; Treufeld, I.; Schuele, D. E.; Ponting, M.; Langhe, D.; Baer, E.; Zhu, L. Reduction of Ionic Conduction Loss in Multilayer Dielectric Films by Immobilizing Impurity Ions in High Glass Transition Temperature Polymer Layers, ACS Appl. Energy Mater. 2018, 1, 775-782.

(16) Tseng, J.-K.; Tang, S.; Zhou, Z.; Mackey, M.; Carr, J. M.; Mu, R.; Flandin, L.; Schuele, D. E.; Baer, E.; Zhu, L. Interfacial Polarization and Layer Thickness Effect on Electrical Insulation in Multilayered Polysulfone/Poly(vinylidene fluoride) films, Polymer 2014, 55, 8-14.

(17) Feng, J.; Zhang, Z.; Bironeau, A.; Guinault, A.; Miquelard-Garnier, G.; Sollogoub, C.; Olah, A.; Baer, E. Breakup Behavior of Nanolayers in Polymeric Multilayer Systems - Creation of Nanosheets and Nanodroplets, Polymer 2018, 143, 19-27.

(18) Liu, R. Y. F.; Ranade, A. P.; Wang, H. P.; Bernal-Lara, T. E.; Hiltner, A.; Baer, E. Forced Assembly of Polymer Nanolayers Thinner Than the Interphase, Macromolecules 2005, 38, 10721-10727.

(19) Liu, R. Y. F.; Bernal-Lara, T. E.; Hiltner, A.; Baer, E. Polymer Interphase Materials by Forced Assembly, Macromolecules 2005, 38, 4819-4827.

(20) Broseta, D.; Fredrickson, G. H.; Helfand, E.; Leibler, L. Molecular Weight and Polydispersity Effects at Polymer-Polymer Interfaces, Macromolecules 1990, 23, 132-139.

(21) Arabeche, K.; Delbreilh, L.; Adhikari, R.; Michler, G. H.; Hiltner, A.; Baer, E.; Saiter, J.-M. Study of the Cooperativity at the Glass Transition Temperature in PC/PMMA Multilayered Films: Influence of Thickness Reduction from Macro- to Nanoscale, Polymer 2012, 53, 1355-1361.

(22) Casalini, R.; Zhu, L.; Baer, E.; Roland, C. M. Segmental Dynamics and the Correlation Length in Nanoconfined PMMA, Polymer 2016, 88, 133-136.

(23) Pollock, G.; Nazarenko, S.; Hiltner, A.; Baer, E. Interdiffusion in Microlayered Polymer Composites of Polycarbonate and a Copolyester, J. Appl. Polym. Sci. 1994, 52, 163-176.

(24) Khariwala, D. Structure-property Relationships in Multilayered Polymeric System and Olefinic Block Copolymers. Ph.D. Thesis, Case Western Reserve University, Cleveland, USA, 2011.

(25) Schuman, T.; Stepanov, E. V.; Nazarenko, S.; Capaccio, G.; Hiltner, A.; Baer, E. Interdiffusion of Linear and Branched Polyethylene in Microlayers Studied via Melting Behavior, Macromolecules 1998, 31, 4551-4561.

(26) Qiu, H.; Bousmina, M. Determination of Mutual Diffusion Coefficients at Nonsymmetric Polymer/Polymer Interfaces from Rheometry, Macromolecules 2000, 33, 6588-6594.

(27) Zhang, H.; Lamnawar, K.; Maazouz, A. Rheological Modeling of the Mutual Diffusion and the Interphase Development for an Asymmetrical Bilayer Based on PMMA and PVDF Model Compatible Polymers, Macromolecules 2013, 46, 276-299.

(28) Zhang, H.; Lamnawar, K.; Maazouz, A. Fundamental Understanding and Modeling of Diffuse Interphase Properties and Its Role in Interfacial Flow Stability of Multilayer Polymers, Polym. Eng. Sci. 2015, 55, 771-791.

(29) Zhang, H.; Lamnawar, K.; Maazouz, A.; Maia, J. M. A Nonlinear Shear and Elongation Rheological Study of Interfacial Failure in Compatible Bilayer Systems, J. Rheol. 2016, 60, 1-23.

(30) Nishi, T.; Wang, T. Melting Point Depression and Kinetic Effects of Cooling on Crystallization in Poly(vinylidene fluoride)-Poly(methyl methacrylate) Mixtures, Macromolecules 1975, 8, 909-915.

(31) Fetters, L. J.; Lohse, D. J.; Colby, R. H., Chain Dimensions and Entanglement Spacings. In Physical Properties of Polymers Handbook, Mark, J. E., Ed. Springer: New York, 2007; pp 447-454.

(32) Rubinstein, M.; Colby, R. H., Polymer Physics. Oxford University Press: Oxford, 2003.

(33) Lamnawar, K.; Maazouz, A. Rheology and Morphology of Multilayer Reactive Polymers: Effect of Interfacial Area in Interdiffusion/Reaction Phenomena, Rheol. Acta 2008, 47, 383-397.

(34) Price, F. P.; Gilmore, P. T.; Thomas, E. L.; Laurence, R. L. Polymer/Polymer Diffusion. I. Experimental Technique, J. Polym. Sci., Polym. Symp. 1978, 63, 33-44. 
(35) Jones, R. A. L.; Klein, J.; Donald, A. M. Mutual Diffusion in A Miscible Polymer Blend, Nature 1986, $321,161-162$.

(36) Sentmanat, M.; Wang, B. N.; McKinley, G. H. Measuring the Transient Extensional Rheology of Polyethylene Melts Using the SER Universal Testing Platform, J. Rheol. 2005, 49, 585-606.

(37) Ania, F.; Baltá-Calleja, F. J.; Henning, S.; Khariwala, D.; Hiltner, A.; Baer, E. Study of the Multilayered Nanostructure and Thermal Stability of PMMA/PS Amorphous Films, Polymer 2010, 51, 1805-1811.

(38) Jin, Y.; Rogunova, M.; Hiltner, A.; Baer, E.; Nowacki, R.; Galeski, A.; Piorkowska, E. Structure of Polypropylene Crystallized in Confined Nanolayers, J. Polym. Sci., Part B: Polym. Phys. 2004, 42, 3380-3396.

(39) Hirata, Y.; Kotaka, T. Phase Separation and Viscoelastic Behavior of Semicompatible Polymer Blends: Poly(vinylidene fluoride)/Poly (methyl methacrylate) System, Polym. J. 1981, 13, 273-281.

(40) Hahn, B.; Herrmann-Schönherr, O.; Wendorff, J. Evidence for A Crystal-Amorphous Interphase in PVDF and PVDF/PMMA Blends, Polymer 1987, 28, 201-208.

(41) Lu, B.; Lamnawar, K.; Maazouz, A.; Zhang, H. Revealing the Dynamic Heterogeneity of PMMA/PVDF blends: from Microscopic Dynamics to Macroscopic Properties, Soft Matter 2016, 12, 3252-3264.

(42) Saito, H.; Stuehn, B. Exclusion of Noncrystalline Polymer from the Interlamellar Region in Poly(vinylidene fluoride)/Poly(methyl methacrylate) Blends, Macromolecules 1994, 27, 216-218.

(43) Mijovic, J.; Sy, J.-W.; Kwei, T. Reorientational Dynamics of Dipoles in Poly(vinylidene fluoride)/Poly(methyl methacrylate) (PVDF/PMMA) Blends by Dielectric Spectroscopy, Macromolecules 1997, 30, 3042-3050.

(44) Samet, M.; Levchenko, V.; Boiteux, G.; Seytre, G.; Kallel, A.; Serghei, A. Electrode Polarization vs. Maxwell-Wagner-Sillars Interfacial Polarization in Dielectric Spectra of Materials: Characteristic Frequencies and Scaling Laws, J. Chem. Phys. 2015, 142, 194703.

(45) Boersma, A.; van Turnhout, J. Dielectric Study on Size Effects in Polymer Laminates and Blends, J. Polym. Sci., Part B: Polym. Phys. 1998, 36, 2835-2848.

(46) Kremer, F.; Schönhals, A., Broadband Dielectric Spectroscopy. Springer: Berlin-Heidelberg, 2003.

(47) Liu, Q.; Richard, C.; Capsal, J.-F. Control of Crystal Morphology and its Effect on Electromechanical Performances of Electrostrictive P(VDF-TrFE-CTFE) Terpolymer, Eur. Polym. J. 2017, 91, 46-60.

(48) Yin, K.; Zhou, Z.; Schuele, D. E.; Wolak, M.; Zhu, L.; Baer, E. Effects of Interphase Modification and Biaxial Orientation on Dielectric Properties of Poly(ethylene terephthalate)/Poly(vinylidene fluoride-co-hexafluoropropylene) Multilayer Films, ACS Appl. Mater. Interfaces 2016, 8, 13555-13566.

(49) Havriliak, S.; Negami, S. A Complex Plane Representation of Dielectric and Mechanical Relaxation Processes in Some Polymers, Polymer 1967, 8, 161-210.

(50) Nakagawa, K.; Ishida, Y. Annealing Effects in Poly(vinylidene fluoride) as Revealed by Specific Volume Measurements, Differential Scanning Calorimetry, and Electron Microscopy, J. Polym. Sci., Part B: Polym. Phys. 1973, 11, $2153-2171$.

(51) Neagu, E.; Pissis, P.; Apekis, L. Electrical Conductivity Effects in Polyethylene Terephthalate Films, J. Appl. Phys. 2000, 87, 2914-2922.

(52) Lu, H.; Zhang, X.; Zhang, H. Influence of the Relaxation of Maxwell-Wagner-Sillars Polarization and DC conductivity on the Dielectric Behaviors of Nylon 1010, J. Appl. Phys. 2006, 100, 054104.

(53) Kramer, E. J.; Green, P.; Palmstrøm, C. J. Interdiffusion and Marker Movements in Concentrated Polymer-Polymer Diffusion Couples, Polymer 1984, 25, 473-480.

(54) Kim, J. K.; Han, C. D. Polymer-Polymer Interdiffusion during Coextrusion, Polym. Eng. Sci. 1991, 31, $258-269$.

(55) Song, J.; Baker, A. M.; Macosko, C. W.; Ewoldt, R. H. Reactive Coupling between Immiscible Polymer Chains: Acceleration by Compressive Flow, AlChE J. 2013, 59, 3391-3402.

(56) Dealy, J. M.; Larson, R. G., Structure and Rheology of Molten Polymers. Hanser Gardner Publications: Cincinnati, 2006.

(57) Liu, G.; Sun, H.; Rangou, S.; Ntetsikas, K.; Avgeropoulos, A.; Wang, S.-Q. Studying the Origin of “Strain Hardening”: Basic Difference between Extension and Shear, J. Rheol. 2013, 57, 89-104. 
(58) Mekhilef, N.; Hedhli, L.; Moyses, S. Effect of Rheological Strain Hardening on Extrusion Blown Film of Polyvinylidene Fluoride, J. Plast. Film Sheet. 2007, 23, 203-219.

\section{Graphical abstract}

$\begin{array}{cc}\text { A } & \\ \text { Interphase } & I_{1} \\ \mathrm{~B} & I_{2} \\ \mathrm{~A} & I_{1} \\ \mathrm{~B} & I_{3} \\ \mathrm{~A} & I_{1} \\ \mathrm{~B} & I_{2} \\ \mathrm{~A} & I_{1} \\ \mathrm{~B} & \end{array}$

Microlayer

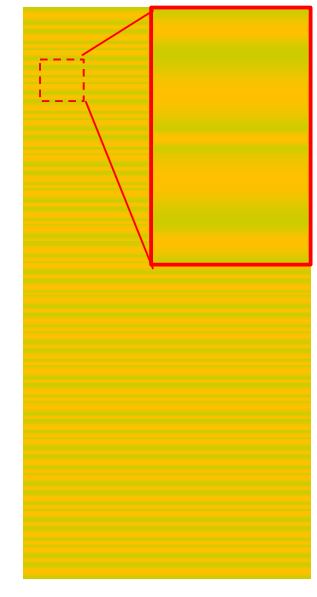

Nanolayer

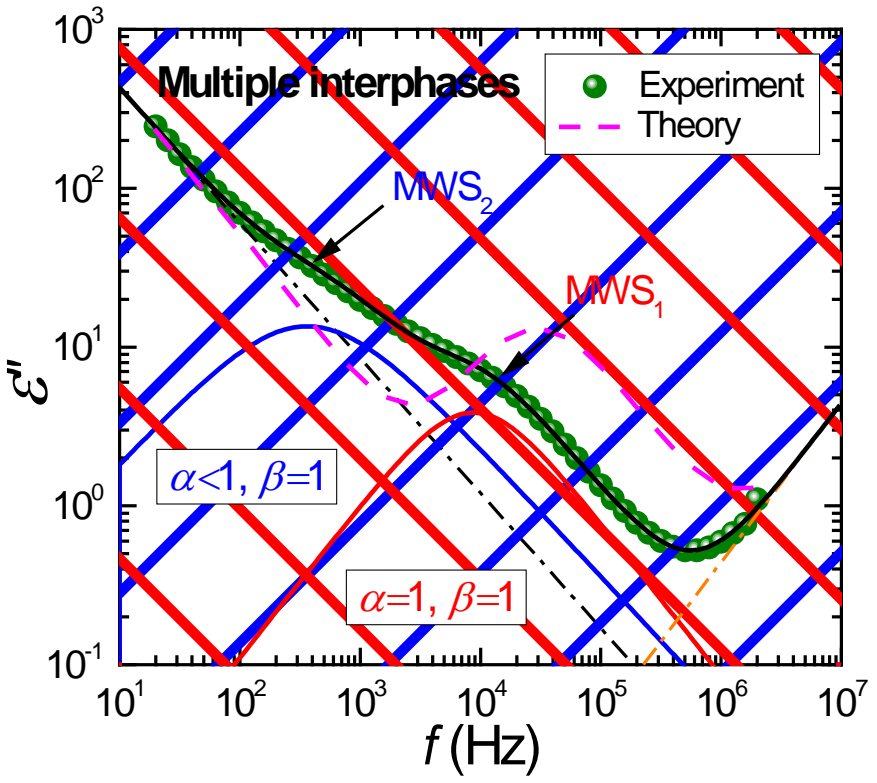

